\title{
Kostenloser ÖPNV: Akzeptanz in der Bevölkerung und mögliche Auswirkungen auf das Mobilitätsverhalten
}

\author{
Mark A. Andor $(\mathbb{D} \cdot$ Lukas Fink • Manuel Frondel $(\mathbb{D} \cdot$ Andreas Gerster $(\mathbb{D} \cdot$ \\ Marco Horvath $(\mathbb{D}$
}

Angenommen: 27. Oktober 2020 / Online publiziert: 20. November 2020

(C) Der/die Autor(en) 2021

Zusammenfassung Der PKW-Verkehr dominiert auch heute noch das Verkehrsaufkommen in Deutschland. Ein häufig diskutierter Vorschlag, um dies zu ändern, lautet, den Öffentlichen Personennahverkehr (ÖPNV) kostenlos anzubieten. Auf Basis einer Erhebung aus dem Jahr 2018, bei der knapp 7000 Haushaltsvorstände des forsaHaushalts-Panels Antworten zu ihrem Mobilitätsverhalten sowie zu ihrer Einstellung gegenüber vieldiskutierten Verkehrsmaßnahmen liefern, untersucht der vorliegende Beitrag die allgemeine Zustimmung zur kostenlosen Nutzung des ÖPNV sowie die möglichen Auswirkungen dieser Maßnahme auf das Mobilitätsverhalten. Die Ergebnisse zeigen eine starke Befürwortung der Maßnahme: Rund $72 \%$ der Antwortenden stimmten für die Einführung eines kostenlosen ÖPNVs. Eine mehrheitliche Zustimmung findet sich in jeder gesellschaftlichen Gruppe, gleich ob männlich oder weiblich, jung oder alt, arm oder reich, sowie in allen politischen Lagern. Nach Einführung eines kostenlosen ÖPNV in Deutschland würden die Befragten die Anzahl ihrer Fahrten mit dem ÖPNV nach eigenen Angaben durchschnittlich um ca. 230\% steigern. Wenngleich es sich um rein hypothetische Antworten handelt und die Ergebnisse daher mit einer gewissen Vorsicht betrachtet werden sollten, deuten diese auf einen relativ starken potenziellen Anstieg der ÖPNV-Nutzung hin. Abschließend diskutieren wir, warum es trotzdem nicht sinnvoll erscheint, den ÖPNV kostenlos anzubieten und welche alternativen Politiken erfolgsversprechender erscheinen.

M. A. Andor $(\bowtie) \cdot$ L. Fink · M. Frondel $\cdot$ M. Horvath RWI - Leibniz-Institut für Wirtschaftsforschung, Essen, Deutschland

E-Mail: andor@rwi-essen.de

M. Frondel

Ruhr-Universität Bochum, Bochum, Deutschland

A. Gerster

Universität Mannheim, Mannheim, Deutschland 
Schlüsselwörter Individualverkehr · Öffentlichen Personennahverkehr ·

Verkehrswende

\title{
JEL-Classification D12 $\cdot$ R48
}

\section{Free public transport: acceptance among the population and possible effects on mobility behavior}

\begin{abstract}
Even today, car traffic still dominates the traffic volume in Germany. A frequently discussed proposal to change this is to offer local public transport free of charge. Based on a survey from 2018, in which nearly 7000 heads of households of the forsa household panel provide answers to their mobility behavior and their attitudes towards much-discussed transport measures, this article examines the general approval of free use of public transport and the possible effects of this measure on mobility behavior. The results show a strong support for the measure: about $72 \%$ of the respondents voted in favor of the introduction of free public transport. The majority agrees in every social group, whether male or female, young or old, poor or rich, and in all political camps. After the introduction of free public transport in Germany, respondents said that they would increase the number of trips they make with public transport by an average of about $230 \%$. Although the answers are purely hypothetical and should therefore be viewed with some caution, they indicate a relatively strong potential increase in public transport use. Finally, we discuss why it still does not seem sensible to offer public transport free of charge and which alternative policies seem more promising.
\end{abstract}

\section{Einleitung}

Der motorisierte Individualverkehr dominiert auch heute noch das Verkehrsaufkommen in Deutschland. So werden $57 \%$ der zurückgelegten Wege und $75 \%$ der zurückgelegten Personenkilometer mit dem Auto bewältigt (BMVI 2018). Mit dieser auf das Auto zentrierten Fortbewegungsweise sind vor allem Stau- und Umweltprobleme verbunden. Letztere werden insbesondere durch lokal und global wirkende Emissionen verursacht. Im Gegensatz zu allen anderen Sektoren sind die Treibhausgasemissionen im Straßenverkehr seit 1990 nicht gesunken, sondern sogar leicht angestiegen (BMU 2019). Technische Verbesserungen, die zu effizienteren und saubereren Motoren führten, wurden konterkariert durch die Zunahme des Güterverkehrs sowie den Anstieg der Anzahl an Kraftfahrzeugen. So stieg die Zahl der Autos in Deutschland zwischen 2007 und 2020 um knapp 16\%, von 41,2 auf 47,7 Mio. (KBA 2020).

Besonders in dicht besiedelten Gebieten und Städten sorgt der Autoverkehr für vielfältige Probleme: er verursacht Lärm, lokale Umweltbelastungen in Form von Stickoxid- und Feinstaubemissionen (UBA 2019) und überlastet die Verkehrsinfrastruktur (BMVI 2017). So wird der öffentliche Raum durch parkende Autos stark eingeschränkt, vor allem in Innenstädten. Zur Vermeidung von immer häufiger auftretenden Verkehrsproblemen in Städten empfehlen Ökonomen die Einführung einer Städte-Maut (z.B. Cramton et al. 2018; Frondel 2019; RWI und Stiftung Mercator 
2019). Neben Maßnahmen, die das Auto als Verkehrsmittel unattraktiver machen, wie die Einführung eines Preises für Kohlendioxid (CO2), der ab dem Jahr 2021 den Kraftstoffeinsatz und den damit einhergehenden $\mathrm{CO} 2$-Ausstoß schrittweise verteuert, erscheint es für eine sozial ausgewogene Verkehrsverlagerung und deren Akzeptanz wichtig, die Alternativen zum Auto zu fördern (Agora Verkehrswende 2018).

Ein häufig diskutierter Vorschlag, um den motorisierten Individualverkehr auf den Öffentlichen Personennahverkehr (ÖPNV) zu verlagern, lautet, diesen kostenlos anzubieten. Dieser Vorschlag ist vor allem im Zuge der Debatte über Stickoxidbelastungen in deutschen Innenstädten in den Fokus der Öffentlichkeit gerückt. In mehreren europäischen Städten wird der ÖPNV bereits kostenfrei angeboten, darunter die estnische Hauptstadt Tallin. In Luxemburg, das zu den vier Ländern der Welt mit der höchsten Autodichte pro Einwohner gehört, wurde im März 2020 ein kostenloser ÖPNV eingeführt. Auch in Deutschland steigt die Häufigkeit der Umsetzung dieser Maßnahme. So ist der ÖPNV seit Beginn des Jahres in der Augsburger Innenstadt kostenlos nutzbar. ${ }^{1}$

Auf Basis einer Erhebung aus dem Jahr 2018, bei der knapp 7000 Haushaltsvorstände des forsa-Haushalts-Panels Antworten zu ihrem Mobilitätsverhalten sowie zu ihrer Einstellung gegenüber vieldiskutierten Verkehrsmaßnahmen lieferten, untersucht der vorliegende Beitrag die Zustimmung zur kostenlosen Nutzung des ÖPNV sowie die möglichen Auswirkungen dieser Maßnahme auf das Mobilitätsverhalten. Den Ergebnissen nach würde diese Maßnahme stark befürwortet: Rund $72 \%$ der Antwortenden stimmten für einen kostenlosen ÖPNV. Eine mehrheitliche Zustimmung findet sich in allen Alters- und Einkommensgruppen, bei Frauen wie Männern sowie in allen politischen Lagern. Vergleichbare Zustimmungswerte fanden Börjesson et al. (2015) in einer Befragung für die Städte Stockholm, Helsinki und Lyon. Der Aussage, dass es sinnvoll wäre, den ÖPNV kostenlos anzubieten, um die StraBenüberlastung zu reduzieren, stimmten in diesen Städten zwischen 59 und $74 \%$ der Befragten zu.

Nach Einführung eines kostenlosen ÖPNV in Deutschland würden die Haushaltsvorstände die Anzahl ihrer Fahrten mit dem ÖPNV nach eigenen Angaben durchschnittlich um ca. $230 \%$ steigern. Bei der Einführung von kostenlosen ÖPNV-Angeboten in verschiedenen europäischen Städten ergaben sich teilweise noch deutlich höhere Nutzungsraten, als es die Erhebungsergebnisse andeuten. So verachtfachte sich die Anzahl der Fahrgäste nach Einführung der kostenlosen Busnutzung in der ostdeutschen Stadt Templin im Jahr 1998 innerhalb eines Jahres. All dies deutet auf einen relativ starken potenziellen Fahrgastanstieg bei kostenloser ÖPNV-Nutzung hin.

Im folgenden Abschnitt wird die Einführung eines kostenlosen ÖPNV aus theoretischer Perspektive skizziert. Nach einer kurzen Erläuterung der Untersuchungsmethodik in Abschn. 3 werden in Abschn. 4 die Ergebnisse in Bezug auf die Einstellung der Befragten zu der Maßnahme präsentiert. Anschließend wird das aktuelle Mobi-

\footnotetext{
${ }^{1}$ Durch die Umsetzung der Maßnahmen stellt sich die Frage der Finanzierung. In Luxemburg sollen die Einnahmeausfälle in Höhe von jährlich 41 Mio.€ durch Steuern ausgeglichen werden (FAZ 2020). In Augsburg wird die Maßnahme die Einnahmen der Stadtwerke Augsburg schätzungsweise um etwa $900.000 €$ pro Jahr verringern (SZ 2020).
} 
litätsverhalten der Haushalte dargestellt und die möglichen Auswirkungen auf die ÖPNV-Nutzung im Falle einer hypothetischen Einführung eines kostenlosen ÖPNV betrachtet. Welche Faktoren die ÖPNV-Nutzung begünstigen, wird in Abschn. 6 mit Hilfe einer OLS-Regression analysiert. Der abschließende Abschnitt fasst die Ergebnisse dieser Analyse zusammen und zieht Schlussfolgerungen.

\section{Theoretische Betrachtungen}

Nach klassischer ökonomischer Theorie entspricht der optimale Preis für die ÖPNVNutzung jenen Kosten, die ein zusätzlicher Passagier durch seine Mitfahrt verursacht. In einer erstbesten Welt würde sich die ÖPNV-Bepreisung daher nach der Auslastung richten und mit den Tageszeiten schwanken. Zu Hauptverkehrszeiten wäre der Preis wesentlich höher als zu Zeiten mit niedriger Auslastung (Storchmann 2003). Außerhalb der Hauptverkehrszeiten fallen die externen Kosten gering aus, in Stoßzeiten kann jedoch jeder weitere Fahrgast den Fahrkomfort der übrigen Fahrgäste nicht unerheblich beeinträchtigen (Sieg 2018): Findet ein Passagier in vollen Bussen und Bahnen einen Sitzplatz, muss ein anderer stehen. Versperrt ein Passagier den Ausgang, verzögern sich das Ein- und Aussteigen und die Haltezeiten erhöhen sich. Für eine optimale Bepreisung ist nicht relevant, dass das eigene Fahrerlebnis (interne Kosten) eines Passagiers schlecht ist. Relevant ist, dass der Fahrkomfort der Mitfahrer verschlechtert wird (externe Kosten). Der optimale Preis würde diese externen Kosten umfassen.

Eine tages- bzw. auslastungsabhängige Bepreisung ist jedoch nur dann wohlfahrtsoptimierend, wenn die Preise aller Mobilitätsalternativen durch die Grenzkosten ihrer Nutzung bestimmt werden. Dies ist jedoch nicht der Fall. So sind die externen Kosten des Pkw-Verkehrs, vor allem die Stau- und die Folgekosten der vom Pkw emittierten Luftschadstoffe, nicht im Preis für Treibstoffe enthalten (Sieg 2018). Daher ist der Preis für die Autonutzung verzerrt. Um diese Verzerrung zu beseitigen und um die volkswirtschaftlichen Kosten des Autoverkehrs verursachergerecht zuzuordnen, wäre in Städten eine nutzungsabhängige Finanzierung der Verkehrsinfrastruktur mit Hilfe einer Städte-Maut sinnvoll (z. B. Cramton et al. 2018; Frondel 2019; RWI und Stiftung Mercator 2019). Damit könnten der öffentliche Raum und die öffentliche Infrastruktur in Städten effizienter genutzt werden; nicht zuletzt ließen sich dadurch die verkehrsbedingten lokalen Schadstoffemissionen effizient bekämpfen (Achtnicht et al. 2018).

Da jedoch nicht zu erwarten ist, dass es eine solche distanz- und belastungsabhängige Mauttechnologie in den nächsten Jahren zu akzeptablen Kosten gibt, ist für den ÖPNV nur eine zweitbeste Lösung möglich: Nach der Second-Best-Theorie wären in dieser ineffizienten Situation geringere Ticketpreise als im First-Best-Optimum angezeigt. Neben den Grenzkosten für die Auto- und ÖPNV-Nutzung hängt die Höhe der Ticketpreise vor allem von der Kreuzpreiselastizität der Autonutzung in Bezug auf Ticketpreiserhöhungen des ÖPNV sowie von der Preiselastizität der ÖPNV-Nachfrage ab (Storchmann 2003), denn neben der Preissensitivität der Nachfrage nach ÖPNV sollte auch die Preisreagibilität des bedeutendsten Substituts zum ÖPNV einen Einfluss auf die ÖPNV-Ticketpreise haben. 
Bei der Höhe der Preissensitivität der ÖPNV-Nachfrage herrscht allerdings große Unklarheit: Die Bandbreite der Schätzwerte für die Preiselastizität liegt laut Literatur zwischen -0,009 und -1,3; bei einem Mittelwert von -0,4 (Holmgren 2007). Wird der ÖPNV-Preis um $1 \%$ erhöht, würde die Nachfrage nach ÖPNV demnach im Durchschnitt um 0,4\% zurückgehen. Die langfristige Kreuzpreiselastizität der Autonutzung in Bezug auf ÖPNV-Ticketpreise beziffert Litman (2019) auf Grundlage der Literatur mit etwa 0,3 bis 0,4. Die Schätzwerte für diese Elastizitäten variieren jedoch stark in Abhängigkeit von Faktoren wie den sozioökonomischen Eigenschaften und den Charakteristika der regionalen Umgebung (Litman 2019; Holmgren 2007; Paulley et al. 2006). Aufgrund großer Bandbreiten für die Elastizitäten und der Unbestimmtheit der Höhe der Grenzkosten ist der optimale ÖPNV-Preis auf empirischer Basis nur sehr schwer bestimmbar. Ein ÖPNV-Preis von null, das heißt ein kostenloses ÖPNV-Angebot, würde allerdings kaum die Second-Best-Lösung reflektieren (Storchmann 2003).

Aus politischer Perspektive hätte die Umsetzung eines kostenlosen ÖPNV-Systems gewisse Reize (Fearnley 2013). Erstens wären mit dieser Maßnahme nicht unerhebliche, politisch erwünschte Umverteilungseffekte verbunden, denn dadurch würden manche Bevölkerungsgruppen begünstigt, etwa einkommensschwache Haushalte und Jugendliche. Zweitens könnten dadurch umweltpolitische Ziele verfolgt werden, indem der Umstieg auf den ÖPNV attraktiver würde. Drittens soll ein kostenloser ÖPNV dazu beitragen, die Effizienz in der Verkehrsinfrastruktur zu erhöhen. So bestünde bei einem Umstieg vom Auto auf den ÖPNV ein geringerer Bedarf an Straßenausbau und auch die Stauprobleme würden weniger werden.

Das Erreichen dieser politischen Ziele ist allerdings davon abhängig, in welchem Umfang vom Auto auf den ÖPNV umgestiegen wird. So waren in der belgischen Stadt Hasselt und der estnischen Hauptstadt Tallinn durch die Einführung eines kostenlosen ÖPNV zwar größtenteils enorme Zuwachsraten zu verzeichnen. Allerdings sind mit Fußgängern und Fahrradfahrern oft die falschen Zielgruppen auf den ÖPNV umgestiegen. Im Falle Templins gaben nur 10-20\% der Befragten an, für die zusätzlichen Fahrten weniger Strecken mit dem Auto zurückzulegen (Storchmann 2001). Van Goeverden et al. (2006) berichten für die belgische Stadt Hasselt eine ähnlich geringe Auswirkung auf den motorisierten Individualverkehr. Lediglich $37 \%$ der zusätzlichen Fahrgäste sind Personen, die zuvor keine Nutzer des ÖPNV waren. Von den Personen, die zuvor den ÖPNV nicht nutzten, ersetzten $43 \%$ Autofahrten durch ÖPNV-Fahrten, die übrigen Umsteiger waren Fahrradfahrer und Fußgänger. Im Fall Tallinn ging der gestiegene Marktanteil des ÖPNV primär zu Lasten des Fußverkehrs. Lediglich $10 \%$ des Anstiegs der ÖPNV-Nutzung werden auf einen Rückgang von Autofahrten zurückgeführt (Cats et al. 2017).

Aus Praxis-Perspektive besteht der gravierendste Nachteil eines fahrscheinfreien Nahverkehrs darin, dass die Einnahmen aus den Ticketverkäufen wegfallen würden. Diese belaufen sich nach Angaben des Deutschen Städte- und Gemeindebundes für alle Verkehrsverbünde in Deutschland auf rund 13 Mrd. $€$ pro Jahr (DStGB 2018). Es ist aus politökonomischen Gründen kaum vorstellbar, dass eine solche Summe auf andere Weise finanziert würde, etwa durch Steuermittel. Schließlich ist der ÖPNV ohnehin bereits in erheblichem Maße von Subventionen abhängig. In einer im Auftrag des VDV, des Städtebunds und der 13 Flächenländer erstellten Studie 
(2009) wird für das Jahr 2025 ein nominaler Finanzierungsbedarf des ÖPNVs durch die öffentliche Hand von 16Mrd. € prognostiziert. Dieser Schätzwert ist jedoch lediglich als unterer Eckwert zu interpretieren. Falls ein ÖPNV zum Nulltarif zu einer deutlich stärkeren Nachfrage führt, würde ein kostenloser ÖPNV aufgrund der erforderlichen Kapazitätserhöhungen mit einem deutlich höheren Finanzierungsbedarf verbunden sein, der zusätzlich zum Entfallen der Ticketeinnahmen entstünde (Gehrke, Groß 2014: 12).

\section{Datenerhebung und Stichprobenbeschreibung}

Die dieser Analyse zugrundeliegende Erhebung wurde von der Stiftung Mercator im Rahmen des Projekts „Mobilitätsdaten für die Verkehrswende“ finanziert und vom Marktforschungsinstitut forsa durchgeführt. Die Konzeption des für die Erhebung verwendeten Fragebogens sowie die empirische Analyse der Daten erfolgte durch das RWI. Die Erhebung dauerte vom 23. April bis zum 12. Juni 2018. Befragt wurden die Haushaltsvorstände des forsa.omninet-Haushaltspanels. Hierbei handelt es sich um ein für die deutschsprachige Bevölkerung ab dem Alter von 14 Jahren repräsentatives Panel. Haushaltsvorstände sind definiert als jene Personen, die typischerweise die weitreichenden finanziellen Entscheidungen für den Haushalt treffen. Die Befragten füllten den Fragebogen mehrheitlich via Internet aus. Haushalte, die über keinen Internetanschluss verfügten, hatten die Möglichkeit, mit Hilfe des Fernsehers an der Befragung teilzunehmen. Für die erfolgreiche Beendigung der

Tab. 1 Verteilung der Stichproben-Haushalte nach Bundesländern und regionale Verteilung der Haushalte in Deutschland laut Mikrozensus im Jahr 2017. (Quelle: Statistisches Bundesamt 2018)

\begin{tabular}{llll}
\hline & $\begin{array}{l}\text { Anzahl teilnehmende } \\
\text { Haushalte }\end{array}$ & $\begin{array}{l}\text { Anteil in Stichprobe } \\
\text { (in \%) }\end{array}$ & $\begin{array}{l}\text { Anteil laut Mikro- } \\
\text { zensus 2017 (in \%) }\end{array}$ \\
\hline Baden-Württemberg & 787 & 10,1 & 12,8 \\
Bayern & 1261 & 16,1 & 15,6 \\
Berlin & 446 & 5,7 & 4,8 \\
Brandenburg & 367 & 4,7 & 3,0 \\
Bremen & 81 & 1,0 & 0,9 \\
Hamburg & 146 & 1,9 & 2,4 \\
Hessen & 544 & 7,0 & 7,4 \\
Mecklenburg-Vorpommern & 144 & 1,8 & 2,0 \\
Niedersachsen & 717 & 9,2 & 9,6 \\
Nordrhein-Westfalen & 1778 & 22,7 & 21,2 \\
Rheinland-Pfalz & 323 & 4,1 & 4,7 \\
Saarland & 78 & 1,0 & 1,2 \\
Sachsen & 409 & 5,2 & 5,2 \\
Sachsen-Anhalt & 222 & 2,8 & 2,8 \\
Schleswig-Holstein & 293 & 3,7 & 3,6 \\
Thüringen & 227 & 2,9 & 2,7 \\
Insgesamt & 7823 & 100,0 & 100,0 \\
\hline
\end{tabular}


Tab. 2 Altersverteilung in der Stichprobe und laut Mikrozensus im Jahr 2017. (Quelle: Statistisches Bundesamt 2018)

\begin{tabular}{lll}
\hline $\begin{array}{l}\text { Im Alter von ... bis unter ... Jah- } \\
\text { ren }\end{array}$ & Anteil in Stichprobe (in \%) & $\begin{array}{l}\text { Anteil laut Mikrozensus } 2017 \\
\text { (in \%) }\end{array}$ \\
\hline Unter 20 & a & 18,7 \\
$20-25$ & 3,8 & 5,5 \\
$25-35$ & 13,2 & 13,0 \\
$35-45$ & 15,1 & 12,3 \\
$45-55$ & 22,1 & 16,0 \\
$55-65$ & 19,5 & 14,2 \\
$65-75$ & 18,2 & 10,1 \\
$75-85$ & 7,1 & 8,3 \\
85 und älter & 0,3 & 2,2 \\
\hline
\end{tabular}

an der Stichprobe sind in der Gruppe der unter 20-Jährigen lediglich 18- und 19-Jährige enthalten, während beim Mikrozensus auch Kinder und Jugendliche in diese Kategorie fallen

Befragung erhielten die Teilnehmer Bonuspunkte, die sie ähnlich einem PaybackSystem in Prämien eintauschen können.

Insgesamt wurden 7823 Haushaltsvorstände befragt, 6812 haben den Fragebogen vollständig ausgefüllt. Die durchschnittliche Bearbeitungszeit betrug ca. 33 min. Die Aufteilung der Teilnehmer-Haushalte auf die Bundesländer stimmt annähernd mit der Verteilung der Haushalte in Deutschland überein, wie sie aus dem Mikrozensus für das Jahr 2017 resultiert (Tab. 1). Lediglich Baden-Württemberg, Brandenburg und Nordrhein-Westfalen weisen größere Abweichungen von bis zu 2,7 Prozentpunkten auf. 52,3\% der Befragten sind männlich, 47,7\% weiblich. Der im Vergleich zur Bevölkerung etwas höhere Anteil männlicher Befragter kann darauf zurückgeführt werden, dass die Befragung explizit an die Haushaltsvorstände gerichtet war.

Die Altersspanne der Befragten reicht von 18-92 Jahren. Die größte Altersgruppe bilden die 45- bis 55-Jährigen. Diese Altersgruppe ist auch in der Bevölkerung am stärksten vertreten (Tab. 2). Obwohl die Bruttostichprobe von forsa repräsentativ ausgewählt ist, ist die Nettostichprobe nicht repräsentativ für Deutschland: Während die Altersgruppen der 35- bis 75-Jährigen überrepräsentiert sind, sind vor allem die unter 20-Jährigen und die 20- bis 25-Jährigen unterrepräsentiert. Diese Diskrepanzen sind u. a. der Tatsache geschuldet, dass Kinder und Jugendliche unter 14 Jahren nicht im forsa-Panel vertreten sind und jüngere Erwachsene seltener Haushaltsvorstände repräsentieren.

Tab. 3 Höchster allgemeinbildender Schulabschluss in der Strichprobe und in der Bevölkerung laut Mikrozensus im Jahr 2017. (Quelle: Statistisches Bundesamt 2018)

\begin{tabular}{lll}
\hline & Anteil in Stichprobe (in \%) & Anteil laut Mikrozensus 2017 (in \%) \\
\hline Ohne Schulabschluss & 0,1 & 4,0 \\
Hauptschulabschluss & 19,3 & 30,1 \\
Mittlere Reife & 36,3 & 29,9 \\
(Fach-)Hochschulreife & 44,3 & 32,3 \\
\hline
\end{tabular}


Unterschiede zur Bevölkerung gibt es auch in Bezug auf andere Merkmale: Ähnlich wie in anderen Studien sind höher Gebildete bei den teilnehmenden Haushalten überrepräsentiert (siehe Andor et al. 2014, 2017a; 2017b, 2018a, 2018b). So besitzen 44,3\% der befragten Haushaltsvorstände die (Fach-)Hochschulreife, während dieser Anteil in der Bevölkerung laut Mikrozensus bei lediglich 34,9\% liegt (Tab. 3). Der Akademikeranteil in der Stichprobe liegt bei 32,3\%, in der Bevölkerung lediglich bei $17,8 \%$.

Ein Vergleich unserer Daten zum Mobilitätsverhalten mit der für die deutsche Alltagsmobilität repräsentativen Erhebung „Mobilität in Deutschland“ (MiD) zeigt sehr ähnliche Anteile der ÖPNV-Nutzer für die der Befragung vorangehende Woche: Dieser Anteil liegt in unserer Befragung bei $25 \%$, in der MiD-Erhebung gaben $13 \%$ der Befragten an, den ÖPNV täglich zu nutzen, $10 \%$ nutzen ihn 1-3 Mal in der Woche.

\section{Einstellung zur Einführung eines kostenlosen ÖPNV}

In der öffentlichen Debatte über Stickoxidbelastungen in deutschen Innenstädten wurden zahlreiche verkehrspolitische Maßnahmen, die diesbezüglich Abhilfe schaffen sollen, kontrovers diskutiert. Hierzu gehören neben Fahrverboten für Dieselfahrzeuge, die bestimmte Abgasnormen nicht erfüllen, auch Maßnahmen wie die Einführung eines kostenlosen ÖPNV. Um die Einstellung der Befragten zu diesen verkehrspolitischen Handlungsmöglichkeiten zu ermitteln, wurden sie gefragt, inwieweit sie solche Maßnahmen befürworten. Die Befragten sollten hierzu ihre Zustimmung oder Ablehnung auf einer Likert-Skala mit 5 Antwortmöglichkeiten angeben: „,1 - starke Ablehnung“, „,2 - eher Ablehnung“, ,,3 - weder Zustimmung noch Ablehnung“, „4 - eher Zustimmung“ und ,,5 - starke Zustimmung“. Im Folgen-

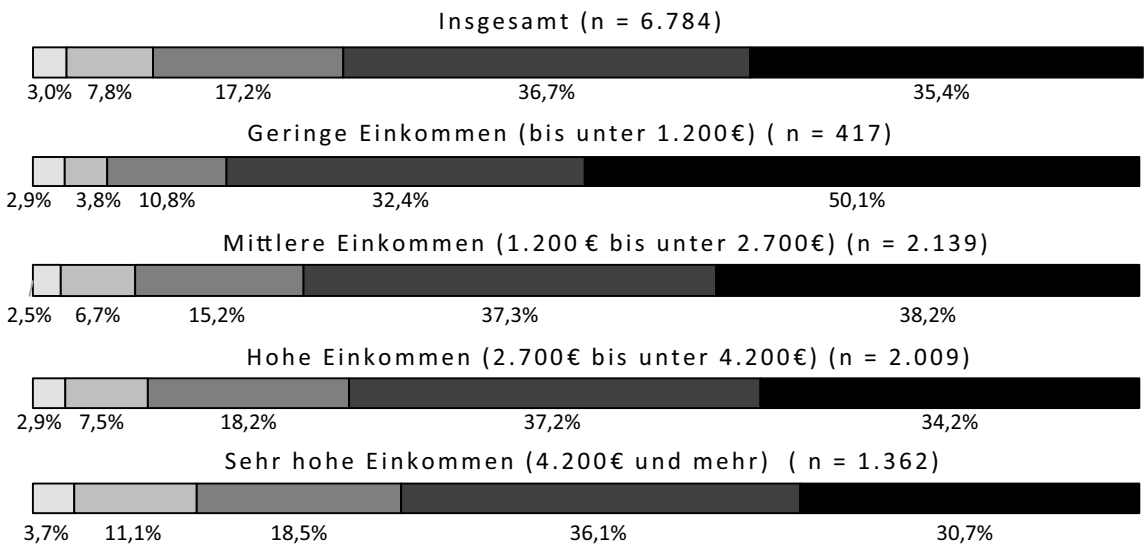

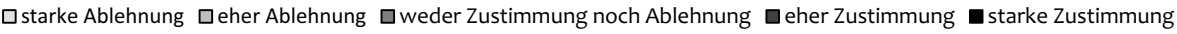

Abb. 1 Ergebnis der Frage: „Bitte markieren Sie, inwieweit Sie persönlich die folgende Maßnahme ablehnen oder befürworten: kostenlose Nutzung von öffentlichen Verkehrsmitteln“ (aufgeteilt nach Einkommensgruppen) 
Ländlicher Raum (<1.000 Einwohner pro $\left.\mathrm{km}^{2}\right)(\mathrm{n}=3.848)$

\begin{tabular}{|c|c|c|c|c|}
\hline \multirow{2}{*}{$2,8 \%$} & $7,6 \%$ & $17,7 \%$ & $39,0 \%$ & $33,0 \%$ \\
\hline & & Städtis & $000 \mathrm{Ein}$ & $.936)$ \\
\hline
\end{tabular}

$\square$ starke Ablehnung $\square$ eher Ablehnung $\square$ weder Zustimmung noch Ablehnung $\square$ eher Zustimmung

starke Zustimmung

Abb. 2 Ergebnisse zur Frage: „Bitte markieren Sie, inwieweit Sie persönlich die folgenden Maßnahmen ablehnen oder befürworten: kostenlose Nutzung von öffentlichen Verkehrsmitteln“ (aufgeteilt nach Art des Wohnorts)

den werden ausschließlich die Ergebnisse zur Einführung eines kostenlosen ÖPNV betrachtet. Dabei werden die Antwortmöglichkeiten 1 und 2 als Ablehnung sowie 4 und 5 als Zustimmung interpretiert und die jeweiligen Anteile addiert.

Mit $72,1 \%$ ist die überwiegende Mehrheit der Befragten für einen kostenlosen ÖPNV (35,4\% ,,starke Zustimmung“ + 36,7\% ,,eher Zustimmung“), rund $17 \%$ stehen der Einführung von kostenlosem ÖPNV neutral gegenüber und knapp $11 \%$ lehnen dies eher oder dezidiert ab (Abb. 1). Damit ist der kostenlose ÖPNV unter allen genannten Maßnahmen jene mit der größten Zustimmung, gefolgt von der Ausweisung reservierter Spuren für Busse und Bahnen auf staubelasteten Straßen mit rund 69\% Zustimmung (Andor et al. 2020a). Die weiteren im Beitrag von Andor et al. (2020a) dargestellten Maßnahmen sind der Ausbau der Infrastruktur für Elektromobilität (Zustimmung: 66,1\%), Fahrverbote für Fahrzeuge, die Feinstaub-/ Schadstoffgrenzwerte überschreiten (Zustimmung: 49,8\%), Ausbau von Fahrradwe-

Die Linke $(n=464)$

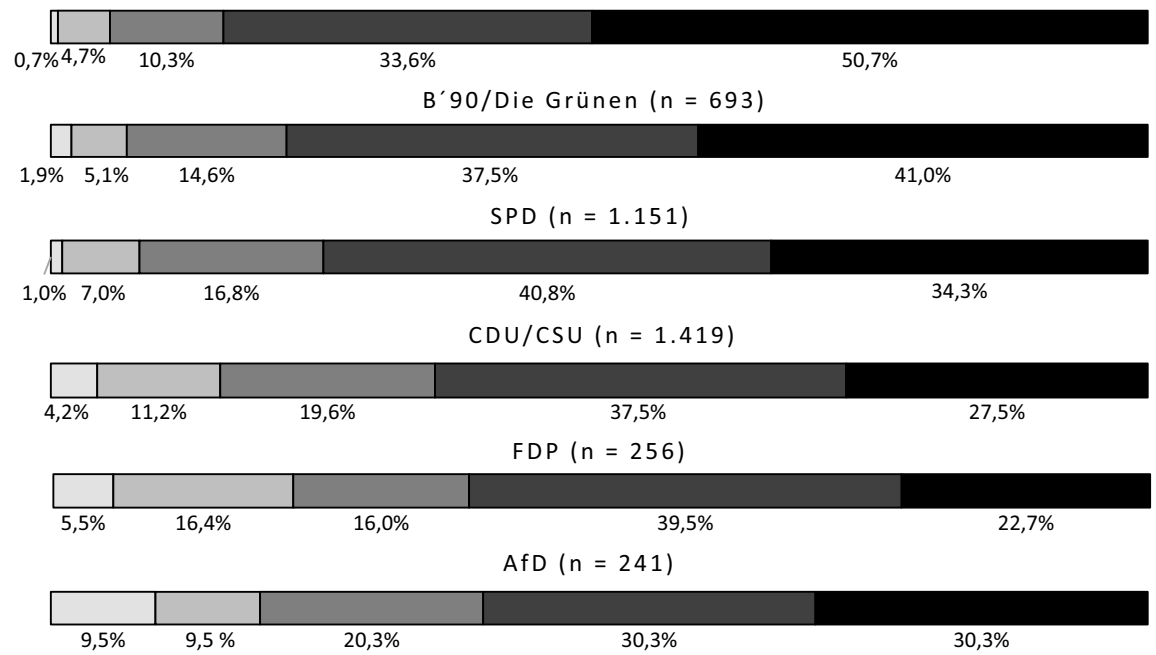

$\square$ starke Ablehnung $\square$ eher Ablehnung $\square$ weder Zustimmung noch Ablehnung $\square$ eher Zustimmung $\mathbf{\square}$ starke Zustimmung

Abb. 3 Ergebnisse zur Frage: „Bitte markieren Sie, inwieweit Sie persönlich die folgenden Maßnahmen ablehnen oder befürworten: kostenlose Nutzung von öffentlichen Verkehrsmitteln“ (aufgeteilt nach Parteineigung der Haushaltsvorstände) 

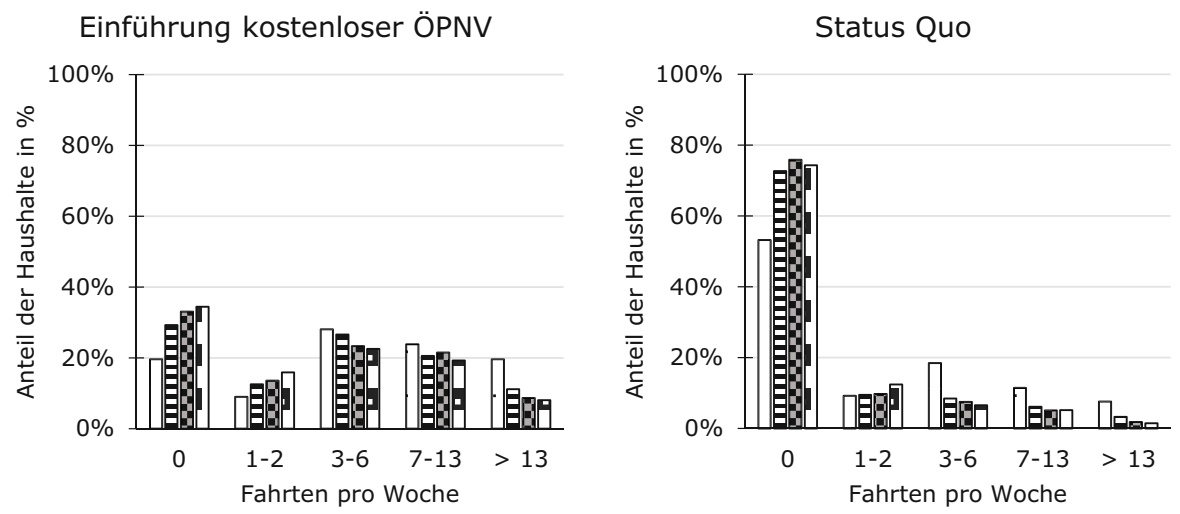

๑geringes Einkommen $\square$ mittleres Einkommen

ahohes Einkommen

asehr hohes Einkommen

Abb. 4 Derzeitige Anzahl der ÖPNV-Fahrten pro Woche und nach Einführung eines kostenlosen ÖPNV nach Einkommensgruppen

gen, wenn nötig auch auf Kosten von Autoparkplätzen (Zustimmung: 49,7\%), Erhöhung der Besteuerung des Verbrauchs von Diesel (Zustimmung: 35,9\%), autofreie Innenstädte (Zustimmung: 34,6\%), ein Verbot von Neuzulassungen von Fahrzeugen mit Verbrennungsmotor ab 2035 (Zustimmung: 27,5\%) und höhere Kosten für das Parken in Innenstädten (Zustimmung: 20,9\%).

Es gibt erhebliche Unterschiede in der Zustimmung zum kostenlosen ÖPNV über Einkommensgruppen hinweg (Abb. 1): Bei Haushalten mit geringem Einkommen ist die Zustimmung mit 82,5\% am höchsten (50,1\% „starke Zustimmung“ + 32,4\% „eher Zustimmung“). Bei steigendem Einkommen nimmt die Zustimmung deutlich ab, bleibt jedoch noch immer hoch. So liegt die Zustimmungsrate in der höchsten Einkommensgruppe bei knapp $67 \%$.

Vergleicht man die Zustimmung zum kostenlosen ÖPNV zwischen Bewohnern des ländlichen und städtischen Raums, zeigen sich - bemerkenswerterweise - kaum Unterschiede (Abb. 2). So sind die Befragten aus dem städtischen Raum mit einer Zustimmungsrate von 72,3\% unwesentlich stärker für die Umsetzung dieser Maßnahme als die Befragten aus dem ländlichen Raum, wo die Zustimmungsrate bei $72,0 \%$ liegt. Zwischen den Bundesländern gibt es ebenfalls keine großen Unterschiede. Lediglich in den Stadtstaaten lehnt ein größerer Anteil die Maßnahme ab als im Bundesdurchschnitt, während in den neuen Bundesländern eine Tendenz zur größeren Zustimmung vorhanden ist (siehe Abb. 5 im Anhang).

Im Hinblick auf die Parteineigung nimmt die Zustimmung zur Einführung eines kostenlosen ÖPNV vom linken zum rechten politischen Spektrum ab (Abb. 3). Die Anhänger der Partei „Die Linke“ stimmen dieser Maßnahme mit 84,3\% (50,7\% ,starke Zustimmung“ + 33,6\% ,eher Zustimmung“) am stärksten zu, gefolgt von Anhängern der Grünen (41,0\% ,starke Zustimmung“" $37,5 \%$,eher Zustimmung“). Trotz eines größeren Anteils an ablehnenden bzw. neutralen Anhängern ist auch bei CDU/CSU, FDP und AfD die große Mehrheit für einen kostenlosen ÖPNV (rund $61-65 \%$ Zustimmung). 


\section{Potenzielle Auswirkungen eines kostenlosen ÖPNV auf die Nutzung}

Ein Teil der Erhebung bestand in der Erfassung des Mobilitätsverhaltens der Haushalte. Die Befragten wurden dazu gebeten, anzugeben, welche Verkehrsmittel sie in den 7 Tagen vor Beantwortung des Fragebogens genutzt haben. In der darauffolgenden Frage sollten die Haushaltsvorstände die Anzahl der Fahrten angeben, die sie innerhalb dieser 7 Tage mit den jeweiligen Verkehrsmitteln zurückgelegt haben (Hin- und Rückfahrten zu einem bestimmten Ort, etwa zum Arbeitsplatz, zählten als einzelne Fahrten.). Die Antworten auf diese beiden Fragen werden im Folgenden genutzt, um den Status Quo der ÖPNV-Nutzung abzubilden.

\subsection{Derzeitige Nutzung des ÖPNV}

In den der Erhebung vorangehenden 7 Tagen wurden von den Befragten im Schnitt 1,6 ÖPNV-Fahrten absolviert (Tab. 4). Personen aus Haushalten mit niedrigem Einkommen nutzen den ÖPNV indessen öfter: Mit 3,4 Fahrten nutzen diese Personen den ÖPNV in etwa doppelt so häufig wie Befragte aus Haushalten mit mittlerem Einkommen. Bei Personen mit hohem oder sehr hohem Haushaltseinkommen fällt die wöchentliche Anzahl der ÖPNV-Fahrten mit einem Durchschnitt von 1,3 etwas niedriger aus als bei mittlerem Einkommen.

Stadtbewohner nutzen den ÖPNV etwa dreimal häufiger als Befragte, die in einer ländlichen Umgebung wohnen. Die Unterschiede zwischen Stadt und Land spiegeln sich auch bei der Betrachtung der einzelnen Bundesländer wider (Tab. 7 im Anhang): Mit im Schnitt 4,2 Fahrten pro Woche und Person in Hamburg und 3,7 Fahrten in Berlin heben sich die zwei großen Stadtstaaten deutlich von den Flächenländern ab. Bei den Flächenländern steht Hessen mit ca. zwei Fahrten pro Woche und Person

Tab. 4 Ergebnisse zu den Fragen: „Wie häufig haben Sie den öffentlichen Nahverkehr in den letzten 7 Tagen genutzt?“ und ,Wie viele Fahrten pro Woche würden Sie typischerweise zusätzlich/weniger mit dem öffentlichen Nahverkehr durchführen, wenn er kostenlos wäre?“

\begin{tabular}{|c|c|c|c|c|c|}
\hline & $\begin{array}{l}\text { Derzeitige } \\
\text { Fahrten }\end{array}$ & $\begin{array}{l}\text { Hypothetische Fahrten } \\
\text { bei kostenlosem ÖPNV }\end{array}$ & $\begin{array}{l}\text { Absolute } \\
\text { Änderung }\end{array}$ & $\begin{array}{l}\text { Relative } \\
\text { Änderung (in \%) }\end{array}$ & $\begin{array}{l}\text { Zahl an Beo- } \\
\text { bachtungen }\end{array}$ \\
\hline Insgesamt & 1,6 & 5,3 & 3,7 & 231 & 6053 \\
\hline $\begin{array}{l}\text { Geringe } \\
\text { Einkommen }\end{array}$ & 3,4 & 7,6 & 4,2 & 124 & 359 \\
\hline $\begin{array}{l}\text { Mittlere } \\
\text { Einkommen }\end{array}$ & 1,7 & 5,7 & 4,0 & 235 & 1826 \\
\hline $\begin{array}{l}\text { Hohe } \\
\text { Einkom- } \\
\text { men }\end{array}$ & 1,3 & 5,1 & 3,8 & 292 & 1794 \\
\hline $\begin{array}{l}\text { Sehr hohe } \\
\text { Einkommen }\end{array}$ & 1,3 & 4,7 & 3,4 & 262 & 1253 \\
\hline $\begin{array}{l}\text { Ländliches } \\
\text { Gebiet }\end{array}$ & 0,8 & 4,0 & 3,2 & 400 & 3338 \\
\hline $\begin{array}{l}\text { Städtisches } \\
\text { Gebiet }\end{array}$ & 2,5 & 6,8 & 4,3 & 172 & 2715 \\
\hline
\end{tabular}


an der Spitze, während das Saarland und Brandenburg mit weniger als einer Fahrt pro Woche die Schlusslichter bilden.

\subsection{Hypothetische Mehrnutzung des ÖPNV}

In zwei weiteren Fragen wurde nach der geplanten Änderung der ÖPNV-Nutzung gefragt, falls dieser kostenlos nutzbar würde. So wurde sich erkundigt, ob die Befragten in diesem Fall planen, den ÖPNV häufiger, genauso häufig oder seltener als momentan zu nutzen. Jene Teilnehmer, die in diesem Fall von ihrer bisherigen Nutzung abweichen wollten, wurden daraufhin gefragt, wie viele Fahrten sie typischerweise zusätzlich oder weniger mit dem ÖPNV wöchentlich zurücklegen würden. Auf Basis der Angaben zur derzeitigen Nutzung lässt sich durch Addition der hinzukommenden bzw. Subtraktion der wegfallenden Fahrten die hypothetische Nutzung des ÖPNV nach Einführung eines kostenlosen ÖPNV feststellen.

Durch einen Vergleich der hypothetischen mit der derzeitigen Nutzung werden im Folgenden die möglichen Auswirkungen einer Einführung eines kostenlosen ÖPNV dargestellt. Im Einklang mit der starken Befürwortung dieser Maßnahme (siehe Abschn. 4) würde sich die Anzahl der ÖPNV-Nutzer deutlich erhöhen: Während nach eigenen Angaben nur etwa $25 \%$ der Erhebungsteilnehmer den ÖPNV in den dem Befragungszeitpunkt vorangehenden 7 Tagen nutzten, würde die hypothetische Nutzungsquote im Falle der Einführung von kostenlosem ÖPNV auf rund $68 \%$ steigen. Durchschnittlich würden 5,3 Fahrten pro Woche und Person mit dem ÖPNV zurückgelegt werden (Tab. 4). Im Vergleich zum derzeitigen Durchschnitt von 1,6 Fahrten entspricht das einer Steigerung von 3,7 Fahrten. Die Zahl der wöchentlichen ÖPNV-Fahrten pro Person würde sich infolge dieser verkehrspolitischen Maßnahme im Mittel mehr als verdreifachen.

Haushalte aus städtischen Regionen würden nach diesen Ergebnissen ihre Nachfrage nach ÖPNV-Fahrten in absoluten Werten stärker erhöhen als Haushalte in ländlichen Umgebungen (Tab. 4). Diese stärkere Steigerung ist zu erwarten, da Bewohner einer städtischen Region typischerweise besser an den ÖPNV angebunden sind als auf dem Land. Die hypothetischen Antworten deuten allerdings auch darauf hin, dass im ländlichen Raum eine deutliche Steigerung der ÖPNV-Nachfrage zu erwarten wäre. Relativ betrachtet steigt die Nachfrage im ländlichen Raum sogar deutlich stärker als in städtischen Regionen. Nach Angaben der Befragten würde sich die durchschnittliche Anzahl von Fahrten um 400\% erhöhen.

Haushalte mit geringen Einkommen würden die Anzahl an ÖPNV-Fahrten mit einer durchschnittlichen Steigerung um 4,2 Fahrten pro Woche am stärksten erhöhen. Bemerkenswert ist, dass auch Befragte mit hohen und sehr hohen Einkommen angeben, die Zahl der ÖPNV-Fahrten pro Woche deutlich steigern zu wollen, im Mittel um 3,8 bzw. 3,4 Fahrten. In Relation zur derzeitigen ÖPNV-Nutzung wäre die Reaktion der Haushalte mit hohen Einkommen am stärksten, die der Haushalte mit geringem Einkommen am schwächsten. Dies ist auf die bereits heute stark ausgeprägte ÖPNV-Nutzung der Haushalte mit geringen Einkommen zurückzuführen.

Aus Abb. 4 wird ersichtlich, dass der Anteil der Haushalte, die den ÖPNV nicht nutzen, deutlich zurückgehen würde, wenn dieser kostenlos wäre. So würde bei den höheren Einkommensgruppen, von denen derzeit über $70 \%$ den ÖPNV in der 
Regel nicht nutzen, der Nichtnutzeranteil auf deutlich unter $40 \%$ sinken. Bemerkenswert ist zudem, dass mit Ausnahme von Personen mit geringem Einkommen die Nutzungsquoten für alle Einkommenskategorien ähnlich ausfallen.

Bei unserer Erhebung handelt es sich um hypothetische Abfragen, bei denen sich die Frage stellt, ob die Angaben auch mit dem tatsächlichen Verhalten übereinstimmen oder eine systematische Unter- oder Überschätzung zu erwarten ist. Untersuchungen auf Basis von hypothetischen Abfragen in anderen Kontexten zeigen Verzerrungen, bspw. bei der Angabe von Zahlungsbereitschaften für öffentliche Güter (z. B. Andor et al. 2017b). Es stellt sich somit die Frage, wie realistisch die ermittelten Ergebnisse sind. Um dies einschätzen zu können, beschreiben wir im Folgenden Erfahrungen mit der Einführung eines kostenlosen ÖPNV aus der Praxis.

Die Erfahrungen verschiedener Städte mit kostenlosen ÖPNV-Angeboten zeigen teilweise deutlich höhere Nutzungsraten auf, als es die Ergebnisse dieser Studie andeuten. Das bekannteste Beispiel aus Deutschland ist die Stadt Templin, wo die Einführung der kostenlosen Busnutzung im Jahr 1998 zur Folge hatte, dass die Anzahl der Fahrgäste von jährlich rund 40.000 im Jahr 1997 massiv anstieg, auf etwas mehr als 350.000 im darauffolgenden Jahr. Dies entspricht mehr als einer Verachtfachung innerhalb eines Jahres. Bis zur Wiederabschaffung der kostenlosen Busnutzung im Jahr 2003 aus Kostengründen stieg die Anzahl der Fahrgäste pro Jahr auf das 15-fache an, auf rund 600.000 im Jahr 2002 (Gehrke und Groß 2014). Die Nahverkehrskosten stiegen für die Stadt Templin von rund 24.000€ im Jahr 1998 auf mehr als $85.000 €$ im Jahr 2000. Um diese Kosten zumindest teilweise durch Einnahmen decken zu können und um der Vollbesetzung der Busse entgegenzuwirken, wurde im Jahr 2003 das kostenlose Busfahren wieder abgeschafft. Daraufhin sank die Zahl der Fahrgäste wieder beträchtlich, blieb aber mit derzeit rund 280.000 deutlich über dem Niveau, das vor der Einführung des ehemals kostenlosen BusTickets herrschte.

Ein weiteres Beispiel ist die belgische Stadt Hasselt, die aufgrund eines erheblichen Anstiegs des motorisierten Individualverkehrs die kostenfreie Nutzung öffentlicher Verkehrsmittel im Jahr 1997 einführte. Die Anzahl der Fahrgäste stieg von etwa 360.000 im Jahr 1996 auf ca. 1,5 Mio. im Jahr der Einführung der Maßnahme (Gehrke und Groß 2014). Bis zum Jahr 2008 hatte sich die Anzahl der Fahrgäste gegenüber dem Jahr 1996 in etwa verzwölffacht.

Die estnische Hauptstadt Tallinn bietet seit dem Jahr 2013 den ÖPNV kostenlos an. Die Anzahl der Fahrten mit dem ÖPNV lag ein Jahr danach lediglich um 14\% höher. Diese vergleichsweise moderate Reaktion wird darauf zurückgeführt, dass es bereits zuvor einen hohen Nutzungsgrad, niedrige ÖPNV-Preise sowie eine gute Anbindung an öffentliche Verkehrsmittel gab (Cats et al. 2017).

\section{Heterogenitätsanalyse: Was begünstigt die ÖPNV-Nutzung?}

In diesem Abschnitt soll mit Hilfe von Regressionsanalysen nach der Methode der kleinsten Quadrate untersucht werden, welche Personen besonders stark auf die Einführung eines kostenlosen Nahverkehrs reagieren würden. Zuerst werden dazu die Einflussfaktoren der ÖPNV-Nutzung der Befragten in den der Erhebung voran- 
gegangenen 7 Tagen analysiert (Tab. 5). Die erklärenden Variablen, die in Tab. 8 im Anhang definiert sind, bestehen aus sozioökonomischen Merkmalen, etwa dem Geschlecht des Haushaltsvorstandes oder der Einkommensklasse des Haushalts. Zusätzlich werden in der ersten Modellspezifikation Variablen mit Bezug auf das eigene Mobilitätsverhalten berücksichtigt, zum Beispiel die Existenz mehrerer Autos im Haushalt. Darüber hinaus werden Variablen verwendet, die mögliche Barrieren der ÖPNV-Nutzung erfassen, etwa die Anbindung des Haushalts an den ÖPNV und die Taktdichte an der nächstgelegenen Haltestelle.

Der zweiten Spezifikation werden Variablen hinzugefügt, die Ergebnisse aus jenem Teil der Umfrage widerspiegeln, in dem ermittelt wurde, wie die Haushalte das Fahren mit dem Auto sowie mit dem ÖPNV wahrnehmen. Beispielsweise wurde sich danach erkundigt, ob die Befragten Autofahren als Stress empfinden. Die Befragten sollten auf einer 5-Punkt-Likert-Skala angeben, wie sehr sie Aussagen dazu zustimmen.

Die Anzahl an Beobachtungen, die zur Schätzung der beiden Spezifikationen verwendet wurden, liegt bei etwas mehr als 3000 (Tab. 5), während knapp 7000 Haushaltsvorstände den Fragebogen vollständig beantwortet haben. Der wesentliche Grund für diese Diskrepanz liegt in der Verwendung zweier Variablen, die ausschließlich für erwerbstätige Befragte definiert sind und die die Erreichbarkeit des Arbeitsplatzes mit dem Auto bzw. dem ÖPNV erfassen. Es wurden nur die Antworten jener Erhebungsteilnehmer berücksichtigt, die beide Fragen hierzu beantwortet haben.

Wenig überraschend hat der Besitz einer Zeitfahrkarte für den ÖPNV (z.B. Jahres-, Monats- oder Semesterticket) einen positiven Effekt auf die derzeitige ÖPNV-Nutzung (Tab. 5). Kinder im Haushalt sind der einzige sozioökonomische Faktor, der eine Rolle zu spielen scheint, mit einem statistisch signifikanten negativen Effekt. Negative Korrelationen finden sich auch für Haushalte mit mehreren Autos und bei solchen, für die die Arbeitsstelle gut mit dem Auto zu erreichen ist. Haushalte, die eine höhere Anzahl an Wegen pro Woche aufweisen, legen diese Wege auch häufiger mit dem ÖPNV zurück als andere Haushalte. Darüber hinaus weisen eine hohe Taktung des ÖPNV sowie die gute Erreichbarkeit der Arbeitsstelle mit dem ÖPNV einen positiven Zusammenhang mit der ÖPNV-Nutzung auf.

Bei allen Schätzungen werden mit Hilfe von Dummy-Variablen mögliche Unterschiede im ÖPNV-Angebot zwischen verschiedenen Verkehrsverbünden berücksichtigt. ${ }^{2}$ Die diesbezüglich hier nicht dargestellten Schätzergebnisse deuten darauf hin, dass es keine systematischen Unterschiede zwischen den Verkehrsverbünden gibt. Tab. 9 im Anhang zeigt die Schätzergebnisse ohne VerkehrsverbundDummies. Die Schätzergebnisse sind quasi identisch mit den Ergebnissen in Tab. 5.

Zur Analyse der hypothetischen Auswirkungen einer Einführung eines kostenlosen ÖPNV werden zwei Modellspezifikationen betrachtet (Tab. 6), die im We-

\footnotetext{
2 Die Befragten konnten im Wesentlichen den folgenden 11 Verkehrsverbünden zugeordnet werden und andernfalls der Kategorie Sonstige: Hamburger Verkehrsverbund, Rhein-Main-Verkehrsverbund, Verkehrsverbund Berlin-Brandenburg, Verkehrsverbund Rhein-Ruhr, Münchner Verkehrs- und Tarifverbund, Verkehrs- und Tarifverbund Stuttgart, Verkehrsverbund Bremen \& Niedersachsen, Mitteldeutscher Verkehrsverbund, Verkehrsverbund Mittelsachsen, Großraum-Verkehr Hannover, Aachener Verkehrsverbund.
} 
Tab. 5 Kleinste-Quadrate-Regressionsergebnisse zur derzeitigen Nutzung des ÖPNV

\begin{tabular}{|c|c|c|c|c|}
\hline & \multicolumn{4}{|c|}{ Anzahl der Fahrten mit dem ÖPNV in den vorangegangen 7 Tagen } \\
\hline & Koeffizient & Std. Fehler & Koeffizient & Std. Fehler \\
\hline Weiblich & $-0,10$ & $(0,12)$ & $-0,13$ & $(0,11)$ \\
\hline Alter & $-0,01$ & $(0,03)$ & $-0,02$ & $(0,03)$ \\
\hline Alter $\times$ Alter & 0,0001 & $(0,0003)$ & 0,0002 & $(0,0004)$ \\
\hline Kinder im Haushalt & $-0,38^{* * *}$ & $(0,13)$ & $-0,41^{* * *}$ & $(0,13)$ \\
\hline Hochschulabschluss & 0,17 & $(0,13)$ & 0,16 & $(0,12)$ \\
\hline Geringes Haushalts-Einkommen & 0,06 & $(0,35)$ & $-0,08$ & $(0,34)$ \\
\hline Mittleres Haushalts-Einkommen & 0,11 & $(0,16)$ & 0,14 & $(0,16)$ \\
\hline Hohes Haushalts-Einkommen & 0,09 & $(0,13)$ & 0,12 & $(0,13)$ \\
\hline Städtischer Raum & 0,09 & $(0,16)$ & 0,07 & $(0,16)$ \\
\hline \# Zurückgelegte Wege pro Woche & $0,09^{* * *}$ & $(0,01)$ & $0,09^{* * *}$ & $(0,01)$ \\
\hline Mehr als ein Auto & $-0,51^{* * *}$ & $(0,12)$ & $-0,42^{* * *}$ & $(0,12)$ \\
\hline Kein ÖPNV-Ticket & $-4,61^{* * *}$ & $(0,28)$ & $-4,43^{* * *}$ & $(0,28)$ \\
\hline Entfernung zur Haltestelle & $-0,03$ & $(0,02)$ & $-0,03$ & $(0,02)$ \\
\hline ÖPNV-Taktung: $5-10 \mathrm{~min}$ & $0,90^{* * *}$ & $(0,25)$ & $0,85^{* * *}$ & $(0,25)$ \\
\hline ÖPNV-Taktung: 10-20 min & 0,24 & $(0,20)$ & 0,26 & $(0,20)$ \\
\hline ÖPNV-Taktung: $20-30 \mathrm{~min}$ & 0,13 & $(0,16)$ & 0,08 & $(0,16)$ \\
\hline $\begin{array}{l}\text { ÖPNV-Taktung: seltener als } \\
\text { stündlich }\end{array}$ & 0,07 & $(0,13)$ & 0,06 & $(0,13)$ \\
\hline ÖPNV-Erreichbarkeit der Arbeit & $0,49^{* * *}$ & $(0,05)$ & $0,49^{* * *}$ & $(0,05)$ \\
\hline Auto-Erreichbarkeit der Arbeit & $-0,49^{* * *}$ & $(0,07)$ & $-0,38^{* * *}$ & $(0,07)$ \\
\hline Gut ausgebaute Straßen & 0,04 & $(0,06)$ & 0,08 & $(0,06)$ \\
\hline Parkplatznähe & $-0,08$ & $(0,05)$ & $-0,04$ & $(0,06)$ \\
\hline Autofahren ist Stress & - & - & $0,10^{*}$ & $(0,06)$ \\
\hline Rechtzeitiges Ankommen mit Auto & - & - & $-0,24^{* * *}$ & $(0,07)$ \\
\hline Flexibilität mit dem Auto & - & - & 0,10 & $(0,11)$ \\
\hline Erledigung anderer Dinge im Auto & - & - & $-0,09^{* *}$ & $(0,04)$ \\
\hline Autofahren kostet wenig & - & - & 0,01 & $(0,05)$ \\
\hline Autofahren ist komfortabel & - & - & $-0,16$ & $(0,10)$ \\
\hline Konstante & $5,58^{* * *}$ & $(0,91)$ & $5,93^{* * *}$ & $(1,02)$ \\
\hline Verkehrsverbund-Dummies & $\mathrm{Ja}$ & & $\mathrm{Ja}$ & \\
\hline Zahl an Beobachtungen & 3066 & & 3031 & \\
\hline $\mathrm{R}^{2}$ & 0,463 & & 0,465 & \\
\hline Adj. $\mathrm{R}^{2}$ & 0,457 & & 0,458 & \\
\hline
\end{tabular}

Robuste Standardfehler in Klammern

*zeigt ein Signifikanzniveau von $10 \%$ an, ** ein Signifikanzniveau von 5\%, *** ein Signifikanzniveau von $1 \%$ 
Tab. 6 Kleinste-Quadrate-Regressionsergebnisse zur hypothetischen Mehrnutzung des ÖPNV bei kostenloser Nutzung

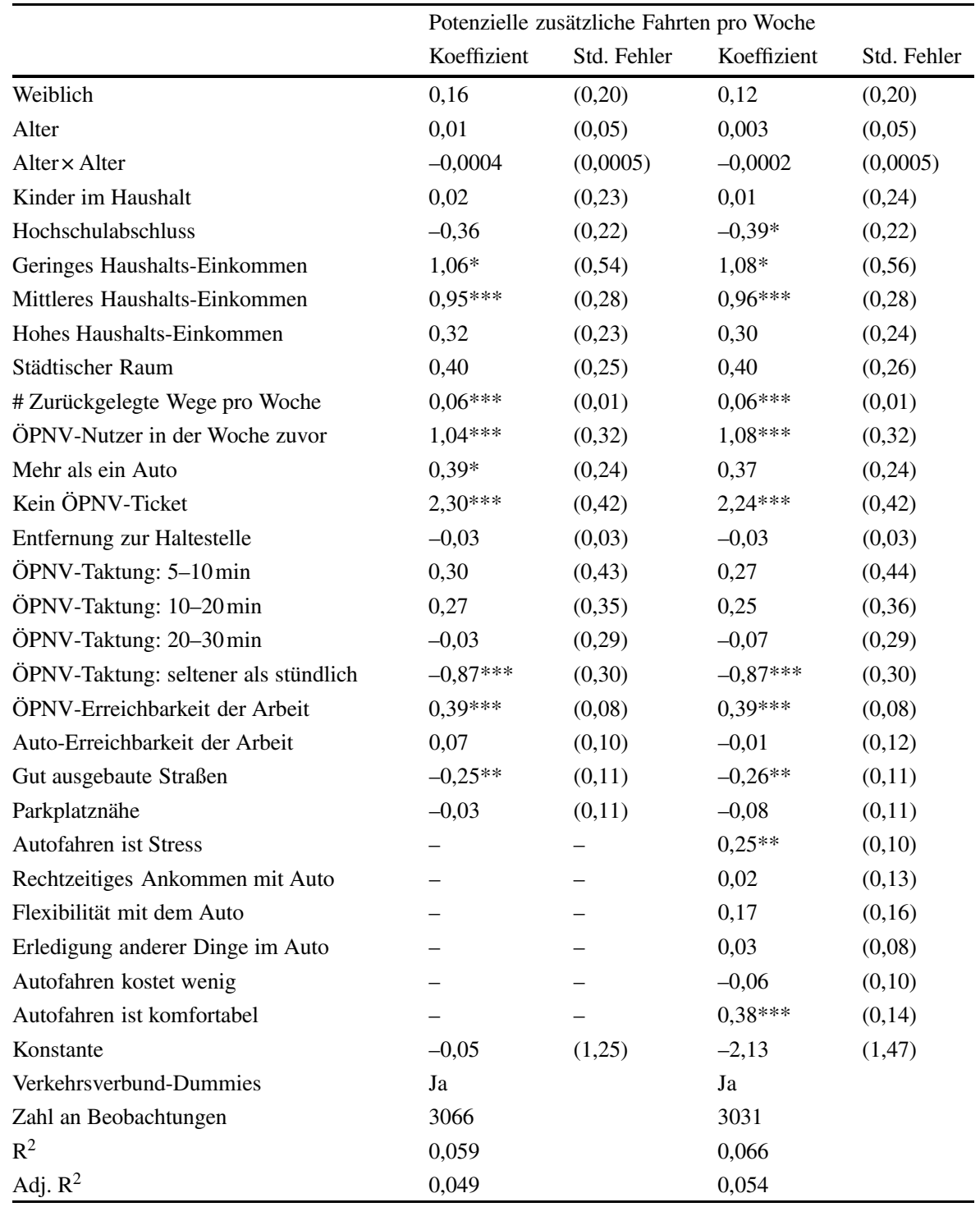

Robuste Standardfehler in Klammern

*zeigt ein Signifikanzniveau von $10 \%$ an, ** ein Signifikanzniveau von 5\%, *** ein Signifikanzniveau von $1 \%$ 
sentlichen dieselben erklärenden Variablen berücksichtigen wie die Schätzungen zur aktuellen ÖPNV-Nutzung. Darüber hinaus wird für die Schätzung der potenziellen Mehrnutzung bei kostenlosem ÖPNV die aktuelle ÖPNV-Nutzung der Befragten berücksichtigt. Die abhängige Variable ist hier für beide Spezifikationen die Mehrnutzung des hypothetisch eingeführten kostenlosen ÖPNV, gemessen in zusätzlichen Fahrten pro Woche. Diese Variable kann bei einer Reduzierung der wöchentlichen Fahrten auch negativ werden. Die Ergebnisse der Schätzungen sind in Tab. 6 dargestellt. $^{3}$

Von den soziodemographischen Variablen spielt nur das Einkommen eine statistisch signifikante Rolle. Diese ist für beide Spezifikationen robust. Im Vergleich zu den Referenz-Haushalten mit sehr hohen Einkommen, die in dieser Regression zur Vermeidung der Dummy-Variablen-Falle weggelassen wurden, steigt die potenzielle ÖPNV-Mehrnutzung nach diesen Regressionsergebnissen mit abnehmenden Einkommen an.

Haushalte, die - unabhängig auf welche Art - viele Wege pro Woche zurücklegen, würden ihre ÖPNV-Nutzung ausweiten, wenn der ÖPNV kostenlos würde, wie der statistisch signifikant positive Schätzwert von 0,06 anzeigt. Befragte, die in den 7 Tagen vor ihrer Befragung den ÖPNV genutzt haben, planen bei Kostenlosigkeit des ÖPNV etwa eine zusätzliche ÖPNV-Fahrt mehr als Befragte, die in der Woche zuvor keine Fahrt mit dem ÖPNV zurückgelegt haben. Der zugehörige Schätzwert liegt in beiden Spezifikationen leicht über dem Wert von 1.

Mit am stärksten würde sich nach diesen Ergebnissen die Einführung der kostenlosen ÖPNV-Nutzung auf diejenigen Befragten auswirken, die derzeit nicht im Besitz eines Tickets sind, mit dem der ÖPNV regelmäßig genutzt werden kann. Diese Personen würden die Zahl der wöchentlichen ÖPNV-Fahrten um mehr als zwei Fahrten ausweiten (siehe Schätzwerte für die Koeffizienten der Dummy-Variablen „Kein ÖPNV-Ticket“ in Tab. 6).

Keinen statistisch signifikanten Einfluss hat hingegen die Entfernung des Haushaltes zur nächstgelegenen Haltestelle. Dies gilt auch für die Taktdichte des ÖPNV, solange diese nicht über einer Stunde liegt. Letzteres wirkt sich negativ auf die ÖPNVNutzung aus. Darüber hinaus ist die hypothetische Mehrnutzung bei kostenlosem ÖPNV umso größer, je größer die Zustimmung zur Aussage „Meine Arbeitsstelle/ Schule/Hochschule ist für mich mit dem ÖPNV gut zu erreichen“ ausfällt.

Wahrnehmungsfaktoren, welche die Mehrnutzung signifikant beeinflussen, sind vor allem die Empfindung von Stress beim Autofahren sowie die Bewertung der Straßeninfrastruktur in der Umgebung des Wohnortes. Je besser Befragte den StraBenzustand und -ausbau bewerten, desto schwächer fällt deren Erhöhung der ÖPNVFahrten aus. Befragte, die das Autofahren als stressig wahrnehmen, würden ihre Fahrten mit dem ÖPNV stärker steigern als andere Autofahrer.

\footnotetext{
3 Tab. 10 im Anhang zeigt die Schätzergebnisse ohne Verkehrsverbund-Dummies. Diese sind praktisch identisch mit den Ergebnissen von Tab. 6.
} 


\section{Zusammenfassung und Schlussfolgerungen}

Vor dem Hintergrund des Überschreitens der EU-Grenzwerte für Stickstoffdioxid in zahlreichen deutschen Innenstädten hat die öffentliche Diskussion über verkehrspolitische Maßnahmen zur Bekämpfung der Verkehrsprobleme in Städten in den vergangenen Jahren an Fahrt aufgenommen. Ein häufig diskutierter Vorschlag lautete in diesem Zusammenhang, den Öffentlichen Personennahverkehr (ÖPNV) kostenlos anzubieten, um den motorisierten Individualverkehr zu verlagern. Basierend auf einer deutschlandweiten Erhebung aus dem Jahr 2018 unter knapp 7000 Haushaltsvorständen des forsa-Haushaltspanels hat dieser Beitrag die hypothetische Einführung eines kostenlosen ÖPNV hinsichtlich der Zustimmung zu dieser Maßnahme und der möglichen Auswirkungen auf die Mobilitätsnachfrage untersucht.

Die Ergebnisse der Erhebung zeigen, dass die Zustimmung zur Einführung eines kostenlosen ÖPNV sehr hoch ist. Mit einer Befürwortung durch $72 \%$ der Antwortenden genießt sie von allen abgefragten Maßnahmen den höchsten Zuspruch und weist eine höhere Akzeptanz auf als beispielsweise der Ausbau der Infrastruktur für Elektromobilität (66\% Zustimmung), Fahrverbote für Fahrzeuge, die Schadstoffgrenzwerte überschreiten (50\% Zustimmung) oder höhere Kosten für das Parken in Innenstädten ( $21 \%$ Zustimmung). Die Zustimmung zur Einführung eines kostenlosen ÖPNV nimmt mit steigendendem Einkommen der Haushalte lediglich moderat ab. Bemerkenswert ist zudem, dass sich bezüglich der Zustimmung zu dieser Maßnahme keine Unterschiede zwischen Haushalten aus ländlichen und städtischen Räumen finden. Die Zustimmung ist am höchsten unter Haushalten, die den linken Parteien des politischen Spektrums zugeneigt sind; dennoch sind auch die Befragten, die tendenziell liberale und rechte Parteien wählen, mehrheitlich für eine Einführung eines kostenlosen ÖPNV (rund 61-65\% Zustimmung).

Den hohen Zustimmungswerten entsprechend würden sich nach Angaben der Haushalte enorme Steigerungsraten für die ÖPNV-Nutzung ergeben, wenn dieser kostenlos angeboten würde. Demnach wäre etwa mit einer Verdreifachung der Nutzung des ÖPNV zu rechnen. Dabei würden Haushalte mit geringen Einkommen die Anzahl an Fahrten absolut betrachtet am stärksten erhöhen, aber auch Haushalte mit mittleren und hohen Einkommen würden ihre ÖPNV-Nutzung ausweiten, relativ gesehen sogar stärker als einkommensschwache Haushalte.

Die in diesem Beitrag vorgenommene Regressionsanalyse stützt im Wesentlichen diese Ergebnisse. Wenig überraschend ist, dass sich nach den Regressionsergebnissen die Einführung der kostenlosen ÖPNV-Nutzung mit am stärksten auf diejenigen auswirkt, die derzeit nicht in Besitz eines Tickets sind, mit dem der ÖPNV regelmäBig genutzt werden kann. Zudem zeigt sich beispielsweise, dass die Erreichbarkeit des Arbeitsplatzes mit dem ÖPNV und der wahrgenommene Stress beim Autofahren positiv mit der Mehrnutzung des ÖPNV korreliert.

Mit dieser Studie gehen, wie bei jeder anderen auch, gewisse Einschränkungen einher. Hier sind vor allem zwei Punkte zu beachten. Erstens wurde bei der Erfragung der Zustimmung zum kostenlosen ÖPNV kein Zielkonflikt beschrieben, da nicht explizit erwähnt wurde, dass die damit verbundenen Kosten auf irgendeine Weise gedeckt werden müssen. Nach Angaben des Deutschen Städte- und Gemeindebundes belaufen sich die Einnahmen der Verkehrsbetriebe in Deutschland auf 
ca. 13 Mrd. $€$ pro Jahr (DStGB 2018). Dieser Betrag müsste auf andere Weise erbracht werden, wenn auf Ticketeinnahmen verzichtet würde. Dies könnte entweder durch Erhöhung der staatlichen Einnahmenseite, bspw. durch Steuererhöhungen, oder durch Kosteneinsparungen an anderer Stelle geschehen, etwa durch die Schließung von öffentlichen Schwimmbädern. Es ist unklar, ob die Akzeptanz für einen kostenlosen ÖPNV noch immer hoch ausfallen würde, wenn den Befragten ein Zielkonflikt deutlich vor Augen geführt würde.

Zweitens erfolgte die Abfrage des potenziellen Mobilitätsverhaltens im Falle eines kostenlosen ÖPNV in hypothetischer Weise. Sowohl die Literatur als auch praktische Erfahrungen zeigen jedoch, dass hypothetische Angaben nicht notwendigerweise dem realen Verhalten entsprechen. Vor dem Hintergrund der empirischen Evidenz zu den Effekten der Einführung eines kostenlosen ÖPNV in einigen Städten erscheinen die ermittelten Werte allerdings nicht unplausibel. So traten in einigen Städten sogar teilweise noch deutlich höhere Nutzungsraten nach deren Einführung auf, als unsere Erhebungsergebnisse es nahelegen würden. Es ist somit bei einem Nulltarif im ÖPNV mit einer deutlichen Ausweitung der Nachfrage zu rechnen. Dies hätte Kapazitätserhöhungen und damit auch eine Erhöhung der Kosten bzw. des Subventionsbedarfs zur Folge (siehe auch Gehrke und Groß 2014).

Es bleibt abzuwarten, welche Erfahrungen sich aus den jüngsten Beispielen zur Einführung eines kostenlosen ÖPNV ergeben, etwa zu Beginn des Jahres 2020 in Augsburg. In jedem Fall wäre es ratsam, solche Maßnahmen von Beginn an durch die Wissenschaft begleiten zu lassen, so wie dies bei der Einführung von Städte-Mauts in skandinavischen Städten geschehen ist (Börjesson et al. 2015). Dadurch ließe sich von neutraler Seite mit relativ hoher Genauigkeit feststellen, welche Effekte die Einführung einer kostenlosen ÖPNV-Nutzung tatsächlich zeitigt.

Abschließend soll hier die Frage aufgeworfen werden, ob ein durchweg kostenloses ÖPNV-Angebot die richtige verkehrspolitische Maßnahme wäre, um die nicht zuletzt aus umweltpolitischer Sicht gewünschte Verlagerung des Individualverkehrs auf den ÖPNV zu intensivieren. Diese Verlagerung ist davon abhängig, in welchem Umfang vom Auto auf den ÖPNV umgestiegen wird. Die bisherigen Erfahrungen in einzelnen Städten zeigen, dass zwar die Nutzung des ÖPNV stark zunimmt, dieser Zuwachs jedoch vornehmlich durch den Wechsel von Fahrradfahrern und Fußgängern auf den ÖPNV ausgelöst wird, nicht durch eine deutliche Reduktion von Autofahrten (Cats et al. 2017; Storchmann 2001; Van Goeverden et al. 2006). Dass Senkungen des ÖPNV-Preises nur begrenzt zur Verringerung des Autofahrens beitragen, zeigen auch Fearnley et al. (2017) in einer Meta-Analyse. Basierend auf 83 Studien ergibt sich, dass eine Reduzierung der Ticketpreise um 1\% im Mittel lediglich zu einer Verringerung des Autofahrens von $0,055 \%$ führt.

Als Alternativen zu einem kostenlosen ÖPNV sehen wir vor allem zwei Maßnahmen als vielversprechend an. Erstens könnten Maßnahmen zur stärkeren Wahrnehmung der Kosten des Autofahrens, wie Informationskampagnen und Labels, erfolgversprechender in Bezug auf die Reduktion des Autobesitzes und der Autonutzung sein, denn aktuelle empirische Evidenz deutet darauf hin, dass Autofahrer in Deutschland die Kosten des Autofahrens in der Regel stark unterschätzen (Andor et al. 2020b). 
Zweitens: Um die vielfältigen Probleme des Autoverkehrs in Städten, insbesondere Staus und lokale Umweltbelastungen in Form von Stickoxid- und Feinstaubemissionen, zu lösen, empfehlen viele Ökonomen die Einführung einer Städte-Maut (z. B. Cramton et al. 2018; Frondel 2019; RWI und Stiftung Mercator 2019). Die MautEinnahmen könnten zur Finanzierung des ÖPNV genutzt werden, um damit die Qualität des ÖPNV zu verbessern und gegebenenfalls die Fahrpreise zu reduzieren.

Danksagung Für Anregungen und Kommentare danken wir einer anonymen Gutachterin bzw. einem anonymen Gutachter, Jean-Pierre Bataille, Weert Canzler, Lars Grotewold, Tobias Larysch, Philipp Offergeld und Lisa Ruhrort.

Förderung Wir bedanken uns bei der Stiftung Mercator für die finanzielle Förderung dieser Studie im Rahmen des Forschungsprojekts „Mobilitätsdaten für die Verkehrswende“ (http://www.rwi-essen.de/ forschung-und-beratung/umwelt-und-ressourcen/projekte/393/).

Funding Open Access funding enabled and organized by Projekt DEAL.

Open Access Dieser Artikel wird unter der Creative Commons Namensnennung 4.0 International Lizenz veröffentlicht, welche die Nutzung, Vervielfältigung, Bearbeitung, Verbreitung und Wiedergabe in jeglichem Medium und Format erlaubt, sofern Sie den/die ursprünglichen Autor(en) und die Quelle ordnungsgemäß nennen, einen Link zur Creative Commons Lizenz beifügen und angeben, ob Änderungen vorgenommen wurden.

Die in diesem Artikel enthaltenen Bilder und sonstiges Drittmaterial unterliegen ebenfalls der genannten Creative Commons Lizenz, sofern sich aus der Abbildungslegende nichts anderes ergibt. Sofern das betreffende Material nicht unter der genannten Creative Commons Lizenz steht und die betreffende Handlung nicht nach gesetzlichen Vorschriften erlaubt ist, ist für die oben aufgeführten Weiterverwendungen des Materials die Einwilligung des jeweiligen Rechteinhabers einzuholen.

Weitere Details zur Lizenz entnehmen Sie bitte der Lizenzinformation auf http://creativecommons.org/ licenses/by/4.0/deed.de. 


\section{Anhang}

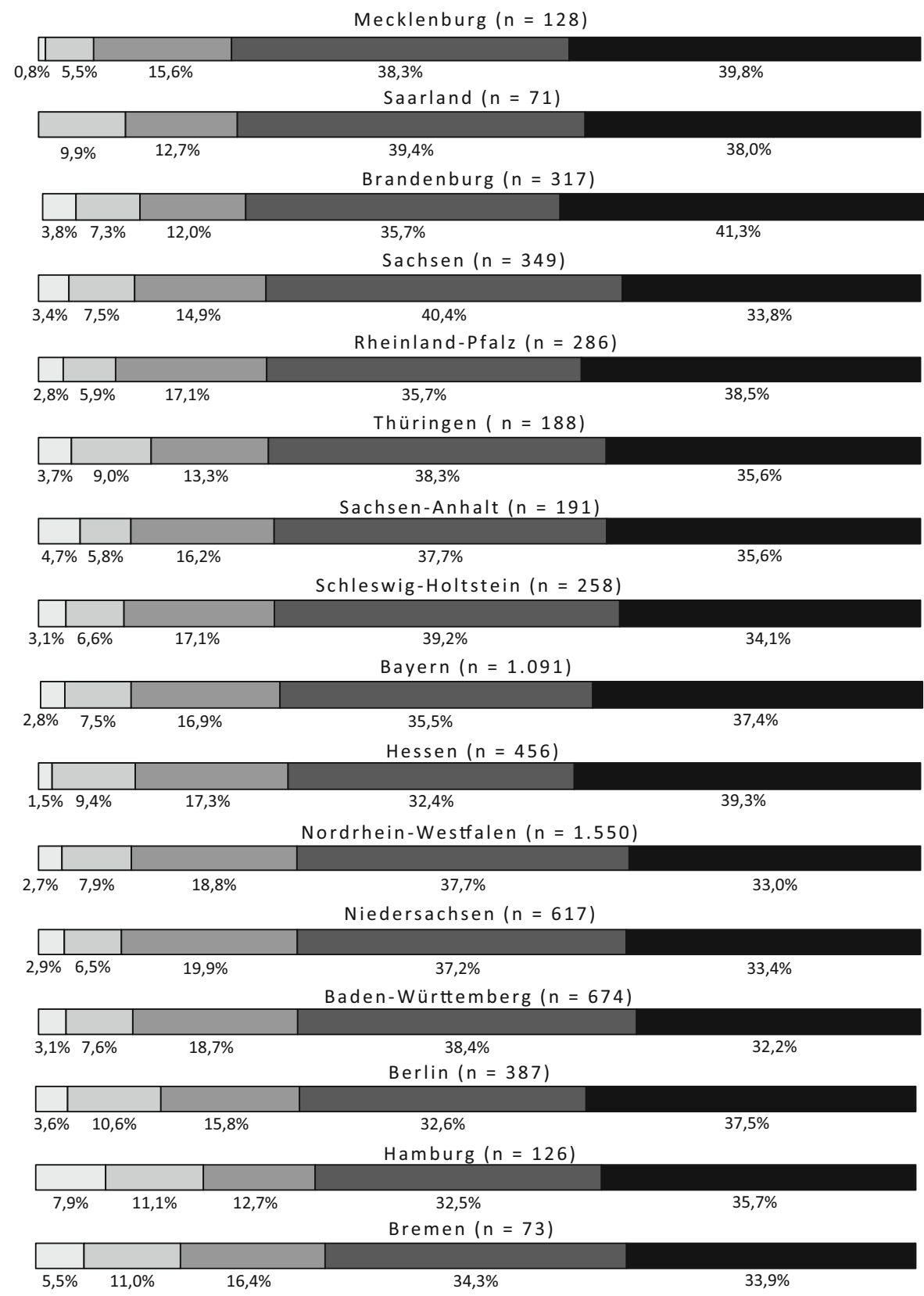

$\square$ starke Ablehnung $\square$ eher Ablehnung $\square$ weder Zustimmung noch Ablehnung $\square$ eher Zustimmung $\mathbf{v}$ starke Zustimmung

Abb. 5 Zustimmung zum kostenlosen ÖPNV als politische Maßnahme nach Bundesländern 
Tab. 7 Bundesländerspezifische Ergebnisse zu den Fragen: „Wie häufig haben Sie den öffentlichen Nahverkehr in den letzten 7 Tagen genutzt?“ und „Wie viele Fahrten pro Woche würden Sie typischerweise zusätzlich/weniger mit dem öffentlichen Nahverkehr durchführen, wenn er kostenlos wäre?“

\begin{tabular}{|c|c|c|c|c|c|}
\hline & $\begin{array}{l}\text { Derzeitige } \\
\text { Fahrten }\end{array}$ & $\begin{array}{l}\text { Hypothetische Fahr- } \\
\text { ten bei kostenlosem } \\
\text { ÖPNV }\end{array}$ & $\begin{array}{l}\text { Absolute } \\
\text { Änderung }\end{array}$ & $\begin{array}{l}\text { Relative } \\
\text { Änderung } \\
\text { (in \%) }\end{array}$ & $\begin{array}{l}\text { Zahl an } \\
\text { Beobach- } \\
\text { tungen }\end{array}$ \\
\hline $\begin{array}{l}\text { Schleswig- } \\
\text { Holstein }\end{array}$ & 1,4 & 5,2 & 3,8 & 280 & 223 \\
\hline Hamburg & 4,2 & 7,3 & 3,1 & 73 & 119 \\
\hline Niedersachsen & 1,1 & 4,8 & 3,7 & 346 & 540 \\
\hline Bremen & 2,0 & 6,0 & 4,0 & 203 & 70 \\
\hline $\begin{array}{l}\text { Nordrhein- } \\
\text { Westfalen }\end{array}$ & 1,3 & 5,2 & 3,9 & 300 & 1376 \\
\hline Hessen & 2,0 & 5,6 & 3,7 & 183 & 404 \\
\hline $\begin{array}{l}\text { Rheinland- } \\
\text { Pfalz }\end{array}$ & 1,1 & 4,8 & 3,6 & 318 & 238 \\
\hline $\begin{array}{l}\text { Baden- } \\
\text { Württemberg }\end{array}$ & 1,6 & 4,9 & 3,3 & 208 & 608 \\
\hline Bayern & 1,6 & 5,0 & 3,4 & 219 & 987 \\
\hline Saarland & 0,9 & 5,3 & 4,3 & 465 & 59 \\
\hline Berlin & 3,7 & 8,3 & 4,6 & 126 & 367 \\
\hline Brandenburg & 1,0 & 4,6 & 3,6 & 380 & 288 \\
\hline $\begin{array}{l}\text { Mecklenburg- } \\
\text { Vorpommern }\end{array}$ & 1,1 & 5,3 & 4,2 & 371 & 109 \\
\hline Sachsen & 1,5 & 5,4 & 3,9 & 259 & 304 \\
\hline $\begin{array}{l}\text { Sachsen- } \\
\text { Anhalt }\end{array}$ & 1,0 & 5,2 & 4,2 & 411 & 171 \\
\hline Thüringen & 1,2 & 4,5 & 3,2 & 263 & 173 \\
\hline
\end{tabular}

Tab. 8 Definitionen der Variablen und zugehörige Mittelwerte

\begin{tabular}{lll}
\hline Variablenname & Variablendefinition & Mittelwert \\
\hline Weiblich & Dummy: 1, wenn Befragter weiblich ist & 0,5 \\
Alter & Alter des Befragten & 51,8 \\
Kinder im Haushalt & Dummy: 1, wenn im Haushalt Kinder unter 14 Jahren leben & 0,2 \\
Hochschulabschluss & Dummy: 1, wenn Befragter tertiären Bildungsabschluss hat & 0,3 \\
Geringes Haushalts- & Dummy: 1, wenn Nettohaushaltseinkommen unter 1200€ & 0,1 \\
Einkommen & liegt & \\
Mittleres Haushalts- & Dummy: 1, wenn Nettohaushaltseinkommen zwischen 1200 & 0,4 \\
Einkommen & und unter 2700€ liegt & \\
Hohes Haushalts- & Dummy: 1, wenn Nettohaushaltseinkommen zwischen 2700 & 0,3 \\
Einkommen & und unter 4200€ liegt & \\
Sehr hohes Haus- & $\begin{array}{l}\text { Dummy: } 1 \text {, wenn Nettohaushaltseinkommen } 4200 € \text { und } \\
\text { halts-Einkommen }\end{array}$ & 0,2 \\
\hline
\end{tabular}


Tab. 8 (Fortsetzung)

\begin{tabular}{|c|c|c|}
\hline Variablenname & Variablendefinition & Mittelwert \\
\hline Städtischer Raum & $\begin{array}{l}\text { Dummy: } 1 \text {, wenn Befragter in einer städtischen Umgebung } \\
\text { lebt }\left(\geqq 1000 \text { Einwohner } / \mathrm{km}^{2}\right)\end{array}$ & 0,4 \\
\hline $\begin{array}{l}\text { \# Zurückgelegte } \\
\text { Wege pro Woche }\end{array}$ & $\begin{array}{l}\text { Anzahl der mit allen Verkehrsmitteln zurückgelegten Strecken } \\
\text { in den vergangenen } 7 \text { Tagen }\end{array}$ & 14,3 \\
\hline ÖPNV-Nutzer & $\begin{array}{l}\text { Dummy: } 1 \text {, wenn Befragter in vergangenen } 7 \text { Tagen den } \\
\text { ÖPNV genutzt hat }\end{array}$ & 0,3 \\
\hline Mehr als ein Auto & Dummy: 1, wenn Befragter mehr als ein Auto besitzt & 0,5 \\
\hline Kein ÖPNV-Ticket & $\begin{array}{l}\text { Dummy 1, wenn Befragter kein Ticket für öffentliche Ver- } \\
\text { kehrsmittel besitzt, mit dem diese regelmäßig genutzt werden } \\
\text { können (Jahres-, Monats- oder Semesterticket) }\end{array}$ & 0,9 \\
\hline $\begin{array}{l}\text { Entfernung Halte- } \\
\text { stelle }(\mathrm{km})\end{array}$ & $\begin{array}{l}\text { Entfernung von der Wohnung des Befragten bis zur nächsten } \\
\text { Haltestelle für öffentliche Verkehrsmittel (in km) }\end{array}$ & 1,1 \\
\hline $\begin{array}{l}\text { ÖPNV-Taktung: } \\
5-10 \mathrm{~min}\end{array}$ & $\begin{array}{l}\text { Dummy: } 1 \text {, wenn von der nächsten Haltestelle öffentliche } \\
\text { Verkehrsmittel ca. alle 5-10 min abfahren }\end{array}$ & 0,1 \\
\hline $\begin{array}{l}\text { ÖPNV-Taktung: } \\
\text { 10-20 min }\end{array}$ & $\begin{array}{l}\text { Dummy: } 1 \text {, wenn von der nächsten Haltestelle öffentliche } \\
\text { Verkehrsmittel ca. alle 10-20 min abfahren }\end{array}$ & 0,2 \\
\hline $\begin{array}{l}\text { ÖPNV-Taktung: } \\
\text { 20-30 min }\end{array}$ & $\begin{array}{l}\text { Dummy: } 1 \text {, wenn von der nächsten Haltestelle öffentliche } \\
\text { Verkehrsmittel ca. alle 20-30 min abfahren }\end{array}$ & 0,2 \\
\hline $\begin{array}{l}\text { ÖPNV-Taktung: } \\
\text { stündlich }\end{array}$ & $\begin{array}{l}\text { Dummy: } 1 \text {, wenn von der nächsten Haltestelle öffentliche } \\
\text { Verkehrsmittel ca. stündlich abfahren }\end{array}$ & 0,2 \\
\hline $\begin{array}{l}\text { ÖPNV-Taktung: } \\
\text { seltener als stündlich }\end{array}$ & $\begin{array}{l}\text { Dummy: } 1 \text {, wenn von der nächsten Haltestelle öffentliche } \\
\text { Verkehrsmittel seltener als stündlich abfahren }\end{array}$ & 0,2 \\
\hline $\begin{array}{l}\text { ÖPNV-Erreichbar- } \\
\text { keit der Arbeit }\end{array}$ & $\begin{array}{l}\text { Erreichbarkeit der Arbeitsstelle/Schule/Hochschule mit dem } \\
\text { ÖPNV }\end{array}$ & 2,4 \\
\hline $\begin{array}{l}\text { Auto-Erreichbarkeit } \\
\text { der Arbeit }\end{array}$ & $\begin{array}{l}\text { Erreichbarkeit der Arbeitsstelle/Schule/Hochschule mit dem } \\
\text { Auto }\end{array}$ & 4,2 \\
\hline $\begin{array}{l}\text { Gut ausgebaute } \\
\text { Straßen }\end{array}$ & $\begin{array}{l}\text { Straßenausbau und -zustand in der Gegend ist zufriedenstel- } \\
\text { lend }\end{array}$ & 3,3 \\
\hline Parkplatznähe & Parkplatz in der Nähe des Wohnorts ist einfach zu finden & 4,0 \\
\hline Autofahren ist Stress & Autofahren ist Stress & 2,2 \\
\hline $\begin{array}{l}\text { Rechtzeitiges An- } \\
\text { kommen }\end{array}$ & $\begin{array}{l}\text { Zuverlässigkeit des Autos in Bezug auf rechtzeitiges Ankom- } \\
\text { men }\end{array}$ & 3,8 \\
\hline Flexibilität mit Auto & Mit dem Auto ist man flexibel & 4,6 \\
\hline $\begin{array}{l}\text { Erledigung anderer } \\
\text { Dinge }\end{array}$ & $\begin{array}{l}\text { Die Möglichkeit der Erledigung anderer Dinge, während des } \\
\text { Autofahrens besteht }\end{array}$ & 2,0 \\
\hline $\begin{array}{l}\text { Autofahren kostet } \\
\text { wenig }\end{array}$ & Autofahren kostet nicht viel & 2,8 \\
\hline $\begin{array}{l}\text { Autofahren ist kom- } \\
\text { fortabel }\end{array}$ & Autofahren ist komfortabel & 4,2 \\
\hline
\end{tabular}


Tab. 9 Kleinste-Quadrate-Regressionsergebnisse zur aktuellen Nutzung des ÖPNV ohne Berücksichtigung von Verkehrsverbund-Dummies

\begin{tabular}{|c|c|c|c|c|}
\hline & \multicolumn{4}{|c|}{ Anzahl der Fahrten mit dem ÖPNV in der vergangenen Woche } \\
\hline & Koeffizient & Std. Fehler & Koeffizient & Std. Fehler \\
\hline Weiblich & $-0,10$ & $(0,11)$ & $-0,13$ & $(0,11)$ \\
\hline Alter & $-0,02$ & $(0,03)$ & $-0,03$ & $(0,03)$ \\
\hline Alter $\times$ Alter & 0,0001 & $(0,0004)$ & 0,0003 & $(0,0004)$ \\
\hline Kinder im Haushalt & $-0,35^{* * *}$ & $(0,13)$ & $-0,39^{* * *}$ & $(0,13)$ \\
\hline Hochschulabschluss & 0,16 & $(0,13)$ & 0,14 & $(0,12)$ \\
\hline Geringes Haushalts-Einkommen & $-0,05$ & $(0,35)$ & $-0,19$ & $(0,34)$ \\
\hline Mittleres Haushalts-Einkommen & 0,06 & $(0,16)$ & 0,10 & $(0,16)$ \\
\hline Hohes Haushalts-Einkommen & 0,09 & $(0,13)$ & 0,11 & $(0,13)$ \\
\hline Städtischer Raum & 0,07 & $(0,15)$ & 0,06 & $(0,15)$ \\
\hline \# Zurückgelegte Wege pro Woche & $0,09^{* * *}$ & $(0,01)$ & $0,09^{* * *}$ & $(0,01)$ \\
\hline Mehr als ein Auto & $-0,56^{* * *}$ & $(0,12)$ & $-0,46^{* * *}$ & $(0,12)$ \\
\hline Kein ÖPNV-Ticket & $-4,66^{* * *}$ & $(0,29)$ & $-4,47^{* * *}$ & $(0,29)$ \\
\hline Entfernung zur Haltestelle & $-0,03$ & $(0,03)$ & $-0,03$ & $(0,02)$ \\
\hline ÖPNV-Taktung: 5-10 min & $0,90^{* * *}$ & $(0,24)$ & $0,83^{* * *}$ & $(0,25)$ \\
\hline ÖPNV-Taktung: 10-20 min & 0,23 & $(0,20)$ & 0,24 & $(0,20)$ \\
\hline ÖPNV-Taktung: 20-30 min & 0,08 & $(0,16)$ & 0,02 & $(0,16)$ \\
\hline ÖPNV-Taktung: seltener als stündlich & 0,09 & $(0,13)$ & 0,08 & $(0,13)$ \\
\hline ÖPNV-Erreichbarkeit der Arbeit & $0,50^{* * *}$ & $(0,05)$ & $0,49^{* * *}$ & $(0,05)$ \\
\hline Auto-Erreichbarkeit der Arbeit & $-0,51^{* * *}$ & $(0,07)$ & $-0,40^{* * *}$ & $(0,07)$ \\
\hline Gut ausgebaute Straßen & 0,06 & $(0,06)$ & $0,10^{*}$ & $(0,06)$ \\
\hline Parkplatznähe & $-0,09$ & $(0,05)$ & $-0,05$ & $(0,06)$ \\
\hline Autofahren ist Stress & - & - & $0,10^{*}$ & $(0,06)$ \\
\hline Rechtzeitiges Ankommen mit Auto & - & - & $-0,25^{* * *}$ & $(0,08)$ \\
\hline Flexibilität mit dem Auto & - & - & 0,10 & $(0,11)$ \\
\hline Erledigung anderer Dinge im Auto & - & - & $-0,09^{* *}$ & $(0,04)$ \\
\hline Autofahren kostet wenig & - & - & 0,01 & $(0,05)$ \\
\hline Autofahren ist komfortabel & - & - & $-0,15$ & $(0,10)$ \\
\hline Konstante & $6,12^{* * *}$ & $(0,91)$ & $6,47^{* * *}$ & $(1,03)$ \\
\hline Verkehrsverbund-Dummies & Nein & & Nein & \\
\hline Zahl an Beobachtungen & 3066 & & 3031 & \\
\hline $\mathrm{R}^{2}$ & 0,455 & & 0,457 & \\
\hline Adj. $\mathrm{R}^{2}$ & 0,452 & & 0,453 & \\
\hline
\end{tabular}

Robuste Standardfehler in Klammern

* zeigt ein Signifikanzniveau von $10 \%$ an, ** ein Signifikanzniveau von 5\%, *** ein Signifikanzniveau von $1 \%$ 
Tab. 10 Kleinste-Quadrate-Regressionsergebnisse zur hypothetischen Mehrnutzung des ÖPNV bei kostenloser Nutzung ohne Berücksichtigung von Verkehrsverbund-Dummies

\begin{tabular}{|c|c|c|c|c|}
\hline & \multicolumn{4}{|c|}{ Potenzielle zusätzliche Fahrten pro Woche } \\
\hline & Koeffizient & Std. Fehler & Koeffizient & Std. Fehler \\
\hline Weiblich & 0,14 & $(0,20)$ & 0,10 & $(0,20)$ \\
\hline Alter & 0,02 & $(0,05)$ & 0,01 & $(0,05)$ \\
\hline Alter $\times$ Alter & $-0,0005$ & $(0,0005)$ & $-0,0004$ & $(0,0005)$ \\
\hline Kinder im Haushalt & 0,02 & $(0,23)$ & 0,02 & $(0,24)$ \\
\hline Hochschulabschluss & $-0,30$ & $(0,21)$ & $-0,34$ & $(0,22)$ \\
\hline Geringes Haushalts-Einkommen & $1,09^{* *}$ & $(0,54)$ & $1,13^{* *}$ & $(0,56)$ \\
\hline Mittleres Haushalts-Einkommen & $0,97^{* * *}$ & $(0,28)$ & $0,98^{* * *}$ & $(0,28)$ \\
\hline Hohes Haushalts-Einkommen & 0,34 & $(0,23)$ & 0,32 & $(0,24)$ \\
\hline Städtischer Raum & $0,46^{*}$ & $(0,25)$ & $0,45^{*}$ & $(0,25)$ \\
\hline \# Zurückgelegte Wege pro Woche & $0,06^{* * *}$ & $(0,01)$ & $0,06^{* * *}$ & $(0,01)$ \\
\hline ÖPNV-Nutzer in der Woche zuvor & $1,02^{* * *}$ & $(0,31)$ & $1,06^{* * *}$ & $(0,32)$ \\
\hline Mehr als ein Auto & $0,41^{*}$ & $(0,24)$ & 0,38 & $(0,24)$ \\
\hline Kein ÖPNV-Ticket & $2,28^{* * *}$ & $(0,43)$ & $2,22^{* * *}$ & $(0,43)$ \\
\hline Entfernung zur Haltestelle & $-0,03$ & $(0,03)$ & $-0,03$ & $(0,03)$ \\
\hline ÖPNV-Taktung: 5-10 min & 0,33 & $(0,44)$ & 0,30 & $(0,44)$ \\
\hline ÖPNV-Taktung: 10-20min & 0,31 & $(0,35)$ & 0,27 & $(0,36)$ \\
\hline ÖPNV-Taktung: 20-30 min & $-0,00$ & $(0,29)$ & $-0,05$ & $(0,29)$ \\
\hline ÖPNV-Taktung: seltener als stündlich & $-0,89^{* * *}$ & $(0,30)$ & $-0,89^{* * *}$ & $(0,30)$ \\
\hline ÖPNV-Erreichbarkeit der Arbeit & $0,39^{* * *}$ & $(0,08)$ & $0,39^{* * *}$ & $(0,08)$ \\
\hline Auto-Erreichbarkeit der Arbeit & 0,08 & $(0,10)$ & $-0,01$ & $(0,12)$ \\
\hline Gut ausgebaute Straßen & $-0,27^{* *}$ & $(0,10)$ & $-0,28^{* * *}$ & $(0,11)$ \\
\hline Parkplatznähe & $-0,02$ & $(0,11)$ & $-0,07$ & $(0,11)$ \\
\hline Autofahren ist Stress & - & - & $0,26^{* *}$ & $(0,10)$ \\
\hline Rechtzeitiges Ankommen mit Auto & - & - & 0,01 & $(0,13)$ \\
\hline Flexibilität mit dem Auto & - & - & 0,18 & $(0,16)$ \\
\hline Erledigung anderer Dinge im Auto & - & - & 0,03 & $(0,08)$ \\
\hline Autofahren kostet wenig & - & - & $-0,05$ & $(0,10)$ \\
\hline Autofahren ist komfortabel & - & - & $0,39^{* * *}$ & $(0,14)$ \\
\hline Konstante & $-0,26$ & $(1,25)$ & $-2,38$ & $(1,47)$ \\
\hline Verkehrsverbund-Dummies & Nein & & Nein & \\
\hline Zahl an Beobachtungen & 3066 & & 3031 & \\
\hline $\mathrm{R}^{2}$ & 0,057 & & 0,064 & \\
\hline Adj. $\mathrm{R}^{2}$ & 0,050 & & 0,055 & \\
\hline
\end{tabular}

Robuste Standardfehler in Klammern

* zeigt ein Signifikanzniveau von $10 \%$ an, $* *$ ein Signifikanzniveau von 5\%, *** ein Signifikanzniveau von $1 \%$ 


\section{Literatur}

Achtnicht, M., Kesternich, M., \& Sturm, B. (2018). Die „Diesel-Debatte“: Ökonomische Handlungsempfehlungen an die Politik. ZEW policy brief 3/2018.

Agora Verkehrswende (2018). Klimaschutz im Verkehr: Maßnahmen zur Erreichung des Sektorziels 2030. https://www.agora-verkehrswende.de/fileadmin/Projekte/2017/Klimaschutzszenarien/Agora_ Verkehswende_Klimaschutz_im_Verkehr_Massnahmen_zur_Erreichung_des_Sektorziels_2030. pdf. Zugegriffen: 16. Mai 2020.

Andor, M. A., Frondel, M., \& Sommer, S. (2014). Klimawandel: Wahrnehmung und Einschätzungen der deutschen Haushalte im Herbst 2012. Zeitschrift für Energiewirtschaft, 38(1), 1-12.

Andor, M. A., Frondel, M., \& Vance, C. (2017a). Germany's Energiewende: a tale of increasing costs and decreasing willingness-to-pay. Energy Journal, 38(Special Issue \#1), 211-228.

Andor, M. A., Frondel, M., \& Vance, C. (2017b). Mitigating hypothetical bias: evidence on the efforts of correctives from a large field study. Environmental and Resource Economics, 68(3), 777-796.

Andor, M. A., Frondel, M., \& Sommer, S. (2018a). Equity and the willingness to pay for green electricity in Germany. Nature Energy, 3(10), 876-881.

Andor, M. A., Schmidt, C. M., \& Sommer, S. (2018b). Climate change, population ageing and public spending: evidence on individual preferences. Ecological Economics, 151, 173-183.

Andor, M. A., Frondel, M., Horvath, M., Larysch, T., \& Ruhrort, L. (2020a). Präferenzen und Einstellungen zu vieldiskutierten verkehrspolitischen Maßnahmen: Ergebnisse einer Erhebung aus dem Jahr 2018. List Forum für Wirtschafts- und Finanzpolitik, 45, 255-280.

Andor, M. A., Gerster, A., Gillingham, K. T., \& Horvath, M. (2020b). Running a car costs much more than people think-stalling the uptake of green travel-Running a car costs much more than people think - stalling the uptake of green travel, Nature, 580(7804), 453-455.

BMU (2019). Treibhausgasemissionen in Deutschland 2018 nach Gas und Kategorie. https://www.bmu. de/fileadmin/Daten_BMU/Download_PDF/Klimaschutz/pi-thg_abbildungen_bf.pdf. Zugegriffen: 16. Mai 2020.

BMVI (2017). Verkehr in Zahlen 2017/2018. https://www.bmvi.de/SharedDocs/DE/Publikationen/G/ verkehr-in-zahlen-pdf-2017-2018.pdf?_blob=publicationFile. Zugegriffen: 16. Mai 2020.

BMVI (2018). Mobilität in Deutschland. Ergebnisbericht. http://www.mobilitaet-indeutschland.de/pdf/ MiD2017_Ergebnisbericht.pdf. Zugegriffen: 16. Mai 2020.

Börjesson, M., Hamilton, C. J., Näsman, P., \& Papaix, C. (2015). Factors driving public support for road congestion reduction policies: congestion charging, free public transport and more roads in stockholm, helsinki and Lyon. Transportation Research Part A: Policy and Practice, 78, 452-462.

Cats, O., Susilo, Y.O., \& Reimal, T. (2017). The prospects of fare-free public transport: evidence from Tallinn. Transportation, 44(5), 1083-1104.

Cramton, P., Geddes, R.R., \& Ockenfels, A. (2018). Set road charges in real time to ease traffic. Nature, $560,23-25$.

DStGB (2018). ÖPNV: Qualität vor Kostenfreiheit. Deutscher Städte- und Gemeindebund. 14.2.2018. https:/www.dstgb.de/dstgb/Homepage/Aktuelles/Archiv/Archiv\%202018/\%C3\%96PNV\%3A\%20Q ualit\%C3\%A4t\%20vor\%20Kostenfreiheit/. Zugegriffen: 16. Mai 2020.

FAZ (2020). Erstes Land der Welt führt kostenlosen ÖPNV ein. https://www.faz.net/aktuell/wirtschaft/ auto-verkehr/luxemburg-fuehrt-kostenlosen-oepnv-ein-16657094.html. Zugegriffen: 16. Mai 2020.

Fearnley, N. (2013). Free fares policies: impact on public transport mode share and other transport policy goals. International Journal of Transportation, 1(1), 75-99.

Fearnley, N., Flügel, S., Killi, M., Gregersen, F. A., Wardman, M., Caspersern, E., \& Toner, J.P. (2017). Triggers of urban passenger mode shift-state of the art and model evidence. Transportation Research Procedia, 26, 62-80.

Frondel, M. (2019). Straßennutzungsgebühren: Eine Lösung zur Vermeidung von Staus? Perspektiven der Wirtschaftspolitik, 20(3), 218-225.

Gehrke, M., \& Groß, S. (2014). Fahrscheinfrei im ÖPNV. IVP-Discussion Paper 03/2014. Berlin: Technische Universität Berlin, Fachgebiet IntegrierteVerkehrsplanung.

van Goeverden, C., Rietveld, P., Koelemeijer, J., \& Peeters, P. (2006). Subsidies in public transport. European Transport, 32, 5-25.

Holmgren, J. (2007). meta-analysis of public transport demand. Transportation Research Part A: Policy and Practice, 41(10), 1021-1035. 
KBA (2020). Jahresbilanz des Fahrzeugbestandes am 1. Januar 2020. https://www.kba.de/DE/Statistik/ Fahrzeuge/Bestand/Jahresbilanz/b_jahresbilanz_inhalt.html?nn=2601598. Zugegriffen: 22. Sept. 2020.

Litman, T. (2019). Transit price elasticities and cross-elasticities. Victoria, Canada: Victoria Transport Policy Institute.

Paulley, N., Balcombe, R., Mackett, R., Titheridge, H., Preston, J., Wardman, M., Shires, J., \& White, P. (2006). The demand for public transport: the effects of fares, quality of service, income and car ownership. Transport Policy, 13(4), 295-306.

RWI, \& Stiftung Mercator (2019). Weniger Staus, Staub und Gestank per sozial ausgewogener StädteMaut: Gemeinsames Plädoyer initiiert von RWI-Leibniz-Institut für Wirtschaftsforschung und der Stiftung Mercator (No. 74). RWI Positionen

Sieg, G. (2018). Kostenloser ÖPNV? Besser gar nicht als falsch einführen. Wirtschaftsdienst, 3, $154-155$.

Statistisches Bundesamt (2018). Bevölkerung und Erwerbstätigkeit. Haushalte und Familien. Ergebnisse des Mikrozensus. Fachserie 1 Reihe 3. Wiesbaden.: DeStatis.

Storchmann, K. (2003). Externalities by automobiles and fare-free transit in Germany-a paradigm shift? Public Transportation, 6(4), 89-105.

Storchmann, K.H. (2001). Nulltarife im öffentlichen Personennahverkehr - ein Paradigmenwechsel? Wirtschaftsdienst, 81(11), 651-657.

SZ (2020). Kostenloser Nahverkehr startet in Augsburg. https://www.sueddeutsche.de/bayern/pilotprojektkostenloser-nahverkehr-startet-in-augsburg-1.4742878. Zugegriffen: 16. Mai 2020.

UBA (2019). Luftbelastung in Ballungsräumen. https://www.umweltbundesamt.de/daten/luft/luftbelastungin-ballungsraeumen\#textpart-1. Zugegriffen: 16. Mai 2020.

Verband Deutscher Verkehrsunternehmen (2009). Finanzierungsbedarf des ÖPNV bis 2025. Köln: Verband Deutscher Verkehrsunternehmen. 